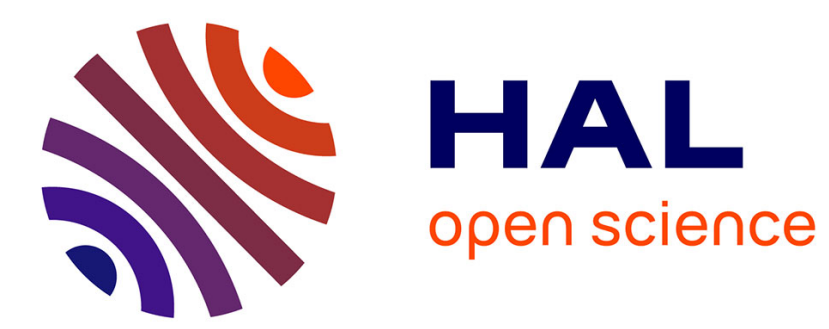

\title{
Philosophie, poésie et musique chez Pléthon
}

\author{
Brigitte Tambrun-Krasker
}

\section{To cite this version:}

Brigitte Tambrun-Krasker. Philosophie, poésie et musique chez Pléthon. 2010. halshs-00460365v2

\section{HAL Id: halshs-00460365 \\ https://shs.hal.science/halshs-00460365v2}

Submitted on 14 Oct 2010

HAL is a multi-disciplinary open access archive for the deposit and dissemination of scientific research documents, whether they are published or not. The documents may come from teaching and research institutions in France or abroad, or from public or private research centers.
L'archive ouverte pluridisciplinaire HAL, est destinée au dépôt et à la diffusion de documents scientifiques de niveau recherche, publiés ou non, émanant des établissements d'enseignement et de recherche français ou étrangers, des laboratoires publics ou privés. 


\title{
Philosophie, poésie et musique chez Pléthon
}

\author{
Brigitte Tambrun-Krasker
}

Le philosophe grec Georges Gémiste surnommé Pléthon (ca. 1360-1452 ou 1454), né et élevé à Constantinople, a suivi le cursus complet des études byzantines, le Trivium et le Quadrivium. A son tour il a dispensé un enseignement; mais suite à une protestation émanant de l'Eglise orthodoxe et portant sur les idées qu'il développait, Pléthon a été envoyé, par l'empereur Manuel II, dans le Péloponnèse, à Mistra près de l'antique Sparte, pour devenir le conseiller politique du jeune Théodore II que le basileus venait de nommer despotês de Mistra.

Or, bien qu'il ait enseigné les disciplines littéraires comme les disciplines scientifiques - et nous en avons la preuve par différents manuels ou traités qui sont conservés ${ }^{1}$ - le savant Gémiste Pléthon porte dans son ouvrage majeur mais secret, le Traité des lois, un jugement très négatif sur la poésie. Il refuse d'y voir la source de la philosophie et de la théologie. Ne fait-il là que reprendre à son compte la distinction classique à Byzance entre la ,sagesse du dehors" (thurathen), autrement dit, les disciplines profanes, et la théologie ?

\section{A la source de toute saine doctrine: les Oracles magiques en vers}

Pour comprendre la démarche intellectuelle de Pléthon, il faut rappeler qu'à son époque, l'Empire des Romains, que nous appelons l'Empire byzantin, est réduit à quelques places fortes et à quelques petites îles ${ }^{2}$. C'est un empire qui se dit toujours universel, mais qui est presque devenu un empire en Idée, sans territoire, c'est-à-dire sans réalisation terrestre.

Le grand problème pour Pléthon qui, installé dans le Péloponnèse, a une famille et deux fils - et même un domaine d'Etat à gérer (une pronoia) ${ }^{3}-$, est de faire en sorte que les Grecs puissent continuer à vivre sur leur territoire menacé par les invasions turques et les occupations latines, et qu'ils puissent transmettre leur patrimoine à la fois familial et hellénique. Or un patrimoine suppose une origine qui est toujours la réalisation dans le temps d'une Idée, et un ancrage dans le sol, sur un territoire. La pensée de Pléthon est donc une pensée de la généalogie, de la régénération, du retour du même, mais il s'agit toujours de relier l'origine et sa réitération, à un lieu terrestre.

Le projet de Pléthon est donc d'accompagner une régénération de l'Empire des Romains, par des réformes politiques, économiques, religieuses et philosophiques. Son programme comporte: premièrement, la reconquête, puis la conservation, des territoires occupés par les Latins et les Turcs, à commencer par le Péloponnèse qu'il faut fortifier au niveau de la muraille d'Hexamilion sur 1'Isthme de Corinthe ${ }^{4}$; deuxièmement, le redémarrage de la politeia hellénique à partir de la région de Sparte (Mistra). Tout ceci nécessite une restructuration de la société et la mise en place d'une politique d'autarcie économique et d'autonomie militaire, mais aussi une nouvelle législation qui elle-même suppose une saine doctrine religieuse et philosophique, et notamment le retour à la philosophie platonicienne. Seule celle-ci permet de comprendre que l'origine est archétypale, et qu'elle doit être réitérée dans le temps et l'espace. Il faut en effet, explique Pléthon, qu'il y ait un accord entre la doctrine religieuse, la philosophie, et les idées qui

\footnotetext{
${ }^{1}$ Voir les références dans Tambrun 2006, 49-50.

${ }^{2}$ Pléthon 1930, 129, 13-17.

${ }^{3}$ Voir les références dans Tambrun 2006, 41.

${ }^{4}$ Sur la reconquête du Péloponnèse au début du XV $\mathrm{XV}^{\mathrm{e}}$ siècle, voir Zakythinos 1953 (reprint 1975), et un bref résumé dans Tambrun 2006, 39-41.
} 
concernent l'organisation politique. Or une saine doctrine religieuse ne peut selon lui se fonder que dans un texte inspiré - mais philosophique et en vers -, qui doit faire concurrence à la Bible. Pléthon considère en effet que le monothéisme - celui des chrétiens, qui est trinitaire, comme celui des Juifs et des Arabes qui pose un dieu seul et unique -, n'est pas favorable à la conservation des empires. Le monothéisme est une forme d'athéisme, comme l'avait déjà souligné l'empereur Julien au IV ${ }^{\mathrm{e}}$ siècle, et déjà - avec humour - un auteur dont Pléthon copie des extraits: Lucien de Samosate ${ }^{5}$. Il s'agit donc de trouver un texte extrêmement ancien qui puisse faire concurrence au Pentateuque, à ce que l'on appelle les „Oracles de Moïse“66. Or, le Ménippe de Lucien de Samosate ne se disait-il pas avec humour, dans l'Icaroménippe, ,accablé par une telle pénurie de Dieux“? Au texte inculte - non philosophique -, du Pentateuque, Pléthon entend substituer une référence concurrente: des „Oracles“ beaucoup plus anciens.

Lucien de Samosate indique la voie sur le ton de la plaisanterie: pour échapper aux contradictions des philosophes qu'il avait fréquentés, Ménippe allait consulter un personnage qui devait le guider dans les enfers pour interroger le devin Tirésias. Or ce guide que Ménippe allait rencontrer à Babylone, était un mage disciple de Zoroastre ${ }^{7}$. Lucien s'amuse en décrivant ce mage sous les traits d'un „chaldéen“, c'est-à-dire d'un magicien qui pratique la goétie. Pléthon pense sans doute qu'il y a un fond, une trace, de vérité, dans ce que dit Lucien: il faut seulement „détordre“ son discours. Mais où retrouver les Oracles des mages disciples de Zoroastre qui pourraient faire concurrence aux „Oracles de Moïse“68 ?

Dans sa jeunesse, Pléthon a vécu en milieu ottoman, à la cour d'Andrinople, chez un Juif lettré nommé Elisha (Elissaios), bon connaisseur des commentaires persans d'Aristote, et donc de traditions orientales sur Zoroastre ${ }^{9}$. Or ce savant met Pléthon sur la piste des „Oracles des mages disciples de Zoroastre“: ce seraient les oracles que l'on appelle improprement „chaldaïques“; ils sont exprimés dans une langue littéraire et non vulgaire, écrits en grec, en hexamètres dactyliques. Pléthon possède une collection de ces Oracles que Michel Psellos a constituée au $\mathrm{XI}^{\mathrm{e}}$ siècle ${ }^{10}$. Il n'ajoute aucun des oracles du même type conservés par les néoplatoniciens, ce qui montre qu'il considère que la collection est complète. Comme ces Oracles ont été selon lui contaminés par le chaldaïsme, c'est-à-dire par la magie vulgaire - dont parle Lucien -, Pléthon retranche tous ceux qui peuvent évoquer la goétie ou des pratiques théurgiques. En outre, il restaure les hexamètres et trouve des solutions métriques qui anticipent celles que Kroll proposera dans son édition des Oracles $^{11}$. Il donne un nouveau titre à la collection: il ne parle jamais d',Oracles chaldaïques“, mais des „Oracles magiques des mages disciples de Zoroastre“"12, indiquant ainsi qu'il faut comprendre le terme de „mage“ au sens de „sage“ comme l'indiquent bien les sources grecques. Enfin, il réorganise complètement la collection transmise par Psellos, de telle sorte que le plan du recueil suit désormais une progression qui va de la psychologie (le devenir de l'âme) à la théologie, en passant par la pratique religieuse.

\footnotetext{
${ }^{5}$ Sur les recueils de textes copiés par Pléthon et conservés à la Biblioteca Marciana de Venise, voir Tambrun 2006, 50.

${ }^{6}$ Voir par exemple le prologue du Dialogue avec Tryphon de Justin; cf. Tambrun 2006, 91-92.

${ }^{7}$ Tambrun 2006, 63.

${ }^{8}$ Tambrun 2006, 92.

${ }^{9}$ Comme l'a montré M. Tardieu, Elisha était un bon connaisseur, sinon un adepte de l'école de Sohrawardî: voir les références dans Tambrun 2006, 36-37 (et note 10); 92-93.

${ }^{10}$ Publiée dans des Places 1971, 162-186 et O’Meara 1989, 126-146.

${ }^{11}$ Voir Tardieu 1987, 141-164.

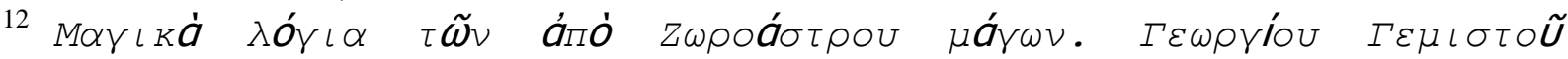

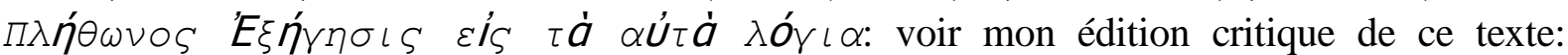
Pléthon 1995.
} 
Cette œuvre philosophique en vers qui a, en réalité, été composée à l'époque du moyenplatonisme, est appelée à concurrencer les „Oracles de Moïse“, car selon les sources grecques, et notamment Plutarque (De Iside 369 D), Zoroastre aurait vécu 5000 ans avant la guerre de Troie $^{13}$. Pythagore, Platon, puis les platoniciens et enfin Georges Gémiste dit Pléthon, seraient les héritiers fidèles de la doctrine des mages perses disciples de Zoroastre. Si l'on fait un calcul car Pléthon est féru de tables d'astronomie ${ }^{14}$, on s'aperçoit que ce Zoroastre ancien est antérieur de 674 ans à la création du monde par le dieu biblique ${ }^{15}$, ce qui disqualifie évidemment la tradition monothéiste d'un seul coup.

Les Oracles magiques dont Pléthon propose un Commentaire ${ }^{16}$ - parce qu'ils sont écrits dans des vers difficiles à comprendre -, présentent évidemment une théologie polythéiste, mais très strictement hiérarchisée: un premier dieu, le Père, un dieu issu du premier (le Nous ou l'Intellect paternel) qui lui-même produit un monde de réalités divines idéales que les Oracles désignent par le terme d',Iynges“ (IU $Y \gamma \in \varsigma$ ) c'est-à-dire de „charmes“.

Or, il faut bien comprendre que cette théologie des Oracles magiques - donc de ce texte en vers -, sert de fondement à tout le système philosophique de Pléthon et de base à toutes ses réformes religieuses et politiques. C'est la matrice de la théologie développée du Traité des lois $^{17}$, ouvrage ouvertement polythéiste de Pléthon, qui n'était connu que d'un petit cercle très restreint de disciples sûrs.

Ainsi, c'est un texte philosophique écrit dans une langue savante très pure - en hexamètres -, et qui serait lui-même la traduction grecque d'une oeuvre de très haute antiquité, exprimée dans une langue très ancienne (celle des Mèdes ou des Perses), qui doit servir de référence ultime et de source à la ,saine doctrine“.

Tout ceci présuppose une certaine idée de la sagesse: les origines de l'humanité sont des origines très savantes; les hommes des temps anciens disposent de l'intégralité de la sagesse; celle-ci n'a pas été acquise progressivement dans l'histoire. Mais en réalité, elle a deux sources: elle se trouve soit sous forme de logia exprimés dans une langue pure, et issus de révélations divines ${ }^{18}$, soit dans l'intellect humain sous forme de sumbola que les dieux ont inscrits en nous ${ }^{19}$. Ces révélations comme ces symboles, sont tous parfaitement rationnels. La sagesse est surtout concentrée; elle est tout entière dans l'origine qui est pleine, et il faut la déployer par le commentaire; il faut aussi la répéter, la réitérer. D'un pays à l'autre, d'un genos à l'autre, d'une langue à l'autre, il existe donc des équivalences linguistiques, qui se réfèrent toutes à la même sagesse divine.

\section{Condamnation de la poésie}

On pourrait s'attendre à ce que Pléthon, qui est platonicien, considère - comme les néoplatoniciens -, les poètes grecs comme des théologiens. Or, ce n'est pas du tout le cas.

Rappelons que les philosophes néoplatoniciens, confrontés à la montée du christianisme qu'ils considéraient comme une doctrine inculte et athée, étaient soucieux de conserver et de sauver

\footnotetext{
${ }^{13}$ Pléthon 1995, 19, 20-22.

${ }^{14}$ Voir Pléthon 1998.

15 Tambrun 2006, 85.

${ }^{16}$ Voir ci-dessus n. 12.

${ }^{17}$ Pléthon 1858.

${ }^{18}$ Pléthon 1858, 32, 5; 42, 14; 86, 14; cf. Pléthon 1989, 392; voir aussi le commentaire de Pléthon sur l'Oracle 11 de sa collection (Pléthon 1995, 2 et 9).

${ }^{19}$ Conformément à l'enseignement des Oracles magiques des mages disciples de Zoroastre: commentaire de l'Oracle 27, dans Pléthon 1995, 16, 6-9.
} 
toute la culture hellénique. Ils devaient contrer les attaques des chrétiens, qui depuis les Pères apologistes, cherchaient à prouver la fausseté de la philosophie, d'une part, en montrant le désaccord des philosophes entre eux et, d'autre part, en expliquant que les récits des poètes sur les dieux grecs étaient immoraux, puisqu'ils comportaient, entre autres horreurs, des meurtres et des adultères. Les philosophes se défendaient d'une part en s'efforçant de montrer qu'il y avait accord, et non dissension, entre les philosophes (Aristote et Platon) ${ }^{20}$, et d'autre part en expliquant que les mythes traditionnels, véhiculés par la culture grecque n'étaient pas monstrueux, car il fallait dépasser leur signification littérale et vulgaire, populaire, pour montrer par une interprétation philosophique, leur sens profond parfaitement pur et saint, concordant avec la philosophie. C'étaient même les invraisemblances des mythes des poètes qui indiquaient qu'ils dissimulaient des vérités et des dogmes divins cachés ${ }^{21}$.

Ainsi, selon Origène ${ }^{22}$, le païen Celse recommandait de prendre pour guides (hodêgoi), les „sages ou philosophes“, entre autres Platon „maitre plus efficace en matière de théologie“, et les anciens poètes, Homère, Orphée, Hésiode. Pour satisfaire au double besoin de concordisme et d'interprétation philosophique des mythes, les néoplatoniciens accordaient et hiérarchisaient entre eux les textes philosophiques: l'œuvre d'Aristote était devenue, dans le cursus néoplatonicien des études, comme une propédeutique à l'œuvre de Platon. Quant aux poètes, Orphée, Homère, Hésiode, ils étaient considérés comme des théologiens qui faisaient autorité, à l'instar de Platon ${ }^{23}$. Les Oracles chaldäques possédaient un statut tout à fait éminent et spécial puisque c'était dans l'étude de cette œuvre de poésie philosophique, contemporaine des médioplatoniciens, et commentée pour la première fois par Porphyre, que culminait le long cursus des études néoplatoniciennes. Les Oracles, les Logia, constituaient même une autorité qui permettait de résoudre les problèmes exégétiques lorsqu'il y avait conflit entre plusieurs interprétations ${ }^{24}$.

Dans l'Essai sur la vie et la poésie d'Homère attribué à Plutarque, et que Pléthon connât ${ }^{25}$, Homère est considéré comme la source de toute la philosophie et même des différentes sectes philosophiques (Pythagore, Platon, Aristote, les stoïciens). Les philosophes, comme les historiens et les orateurs, auraient trouvé dans ses poèmes pour ainsi dire des germes de discours et de toutes sortes de traités (ch. VI). S'il y a parmi les philosophes une grande variété d'opinions, elles sont presque toutes puisées dans Homère (ch. CXXII). Les erreurs d'Epicure ou d'Aristippe proviendraient d'ailleurs d'une mauvaise interprétation de certains de ses textes: ces philosophes se seraient laissés égarer par des discours qu'Homère aurait insérés dans ses poèmes

${ }^{20}$ Pour opérer ce sauvetage, Syrianus, par exemple, proposait une sumphônia, un „Accord entre Orphée, Pythagore, Platon et les Oracles chaldaïques“ (Porphyre étant le premier philosophe néoplatonicien à avoir proposé un commentaire sur les Oracles chaldaïques): voir Proclus 1968, LVII (Introduction).

${ }^{21}$ I. Hadot dans Simplicius 1990, 108-122 et 117.

${ }^{22}$ Contre Celse VII, 41 et 42.

${ }^{23}$ Proclus 1968, LVII (Introduction). Sur l'exégèse d'Homère, voir l'In Rempublicam de Proclus (Kroll 1899, I, 60-205); Friedl 1936; Buffière1956, ${ }^{2}$ 1973, 25-31; 531-540; 541-558.

24 Communication de $\mathrm{Ph}$. Hoffmann, lors du colloque international sur „Les oracles chaldaïques“, dir. Lucia Saudelli et Adrien Lecerf, Paris, EPHE/CNRS (UMR 8584)/ENS, 27 juin 2009 (à paraître).

${ }^{25}$ Dans la Réplique à Scholarios (Pléthon 1989, 476-477), c'est au ch. CXXVIII de l'Essai sur la vie et la poésie d'Homère, que Pléthon se réfère lorsqu'il dit que la théorie du pneuma véhicule de l'âme est attribuée (sans doute à tort) par Plutarque à Aristote; cette source a échappé à B. Lagarde, L.G. Benakis et E.V. Maltese: voir la note 251 de B. Lagarde (dans Pléthon 1989). Pour l'Essai sur la vie et la poésie d'Homère du Pseudo-Plutarque, voir (Pseudo-) Plutarque 1996. 
sans les approuver mais seulement pour s'accorder à certaines circonstances (ch. CL). L'œuvre d'Homère est présentée comme une sorte d'immense encyclopédie à laquelle tous les auteurs des siècles suivants auraient fait des emprunts. Or, l'attitude de Pléthon est très différente. Il prend même le contre-pied de l'Essai sur la vie et la poésie d'Homère.

Rappelons qu'Aristote (Poétique, 1447 b 17-23) préfère appeler „naturalistes“ ( $\varphi v \sigma\llcorner O \lambda o ́ \gamma O\llcorner$ ) plutôt que „poètes“, ceux qui exposent en vers des sujets de médecine ou de physique. Si Homère est appelé un poète, Empédocle qui s'exprime lui aussi en vers, est plutôt un naturaliste. En effet, les textes scientifiques ne sont pas des textes d'imitation, alors que la poésie, selon Aristote, réside avant tout dans l'imitation.

Pléthon dissocie lui aussi les œuvres philosophiques en vers (les Oracles magiques; les Vers d'or des pythagoriciens), de la poésie (Homère, Orphée, etc.), mais pour des raisons un peu différentes. Selon lui, les poètes, comme les „sophistes“ - c'est-à-dire toute l'apologétique chrétienne -, auraient déformé la vérité originelle délivrée, comme on l'a vu, par les dieux ${ }^{26}$.

Dans la doctrine originelle, le sens et la forme sont en parfaite correspondance, en harmonie. La vérité s'exprime dans une langue pure, dans les formes métriques qui conviennent, c'est-à-dire en hexamètres dactyliques ${ }^{27}$. Les poètes conservent bien la forme métrique initiale, mais comme leur but est de divertir et de „charmer“ le public, ils déforment la doctrine originelle en proposant des „fictions“" plaisantes ${ }^{28}$; ils lui font subir des ,distorsions“ $\left(\delta\left\llcorner\propto \sigma \tau \rho \circ \varphi a_{S}\right)^{29}\right.$. Quant aux „sophistes“, c'est-à-dire aux théologiens chrétiens avec qui les poètes sont constamment mis en parallèle, ils font passer pour des révélations originelles, des doctrines complètement corrompues; ce sont des illusionnistes. De plus, il faut comprendre que la langue dans laquelle ils s'expriment est vulgaire: c'est une langue décadente du point de vue de Pléthon qui s'exprime toujours en grec classique (attique): „En effet, les poètes et les sophistes malfaisants, si nombreux à ne donner manifestement ni les uns ni les autres aucune raison valable de ce qu'ils avancent en toute occasion, prétendent les uns comme les autres que c'est par une inspiration des dieux, qui soi-disant leur vient souvent, qu'ils sont instruits de ce qu'ils disent $^{\star 30}$.

Les poètes ne doivent donc pas être considérés comme des guides fiables pour la théologie; et contrairement à ce que tente de démontrer le pseudo-Plutarque dans l'Essai sur la vie et la poésie d'Homère ${ }^{31}$, Homère n'est pas la source de toute la philosophie grecque ${ }^{32}$. Au contraire,

\footnotetext{
${ }^{26}$ De Pléthon, il existe un texte inédit de quelques pages Sur Homère et son Iliade. Il est présent par exemple dans le manuscrit de Le Barbier (coll. privée) dont je possède un microfilm, aux folios 66r-68r, et dans le manuscrit grec C.F.2.11 (folio $240^{\mathrm{rv}}$ ), de la Biblioteca dei Girolamini (Naples).

${ }^{27}$ La perfection de l'hexamètre dactylique est classiquement soulignée par le Pseudo-Plutarque, dans l'Essai sur la vie et la poésie d'Homère (VII) que Pléthon semble avoir en tête, comme par Aristide Quintilien 1963, 47 (Aristide Quintilien 1999, 106).

${ }^{28}$ Pléthon semble ainsi corriger à dessein la thèse de l'Essai sur la vie et la poésie d'Homère (ch. VI) attribué à Plutarque.

${ }^{29}$ Pléthon 1858, 2, traduction [Pellissier] modifiée; voir Couloubaritsis 1997, 139.

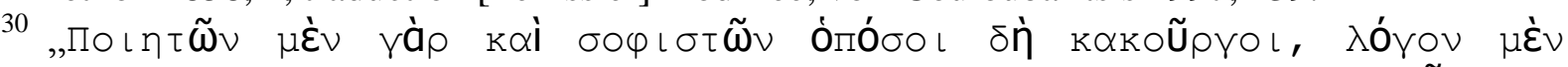

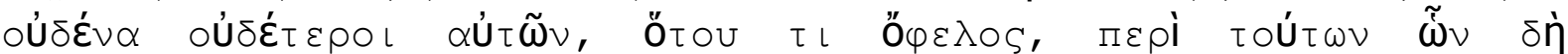

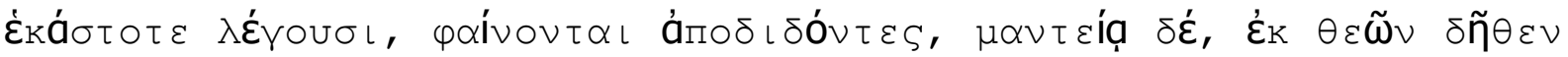

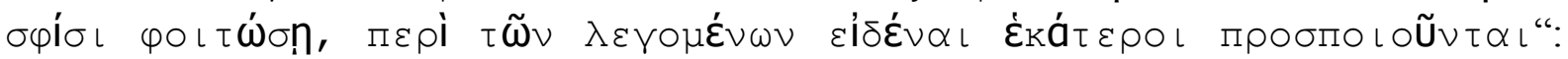
Pléthon 1858, 34

${ }^{31}$ Voir ci-dessus, n. 25.

${ }^{32}$ Sur cette question largement débattue dans l'antiquité tardive, voir Droge 1989.
} 
dans la recherche - entre des opinions contradictoires -, de la doctrine vraie, les poètes comme les „sophistes“ (les chrétiens) sont de bien mauvais guides: „Les poètes, d'une part, sont souvent des flatteurs et ont commerce avec les gens pour le plaisir, mais ils ne se soucient absolument pas de la vérité et de ce qui est le meilleur'“33. Les poètes comme les „,sophistes“, „,rabaissent les affaires des dieux à un niveau plus humain, élèvent les affaires humaines à un niveau plus divin que ce qui convient à l'homme, mettent tout sens dessus dessous, causant le plus grand tort à ceux qui se fient à eux ${ }^{\text {‘634 }}$.

La poésie doit donc être réservée au seul divertissement: „Ainsi les poètes, d'une part, colorant ce qu'ils disent, par l'élégance des noms et du rythme, et séduisant ainsi ceux qui les écoutent, trompent ceux qui ne sont pas capables de faire la part entre le charme des noms et du rythme, et la beauté ou l'insanité de ce qu'ils disent. A vrai dire, le problème pour eux ne serait pas tant de persuader, mais seulement de charmer les auditeurs, qu'ils les persuadent ou non; mais ils semblent agir sur certaines personnes plus qu'ils ne le voudraient ${ }^{t 35}$.

En réalité, à l'époque de Pléthon, personne n'est dupe, de la théologie homérique ! La poésie est clairement considérée comme faisant partie de la „sagesse du dehors“(de la sagesse profane). Le but de Pléthon est donc ici de dénoncer la théologie chrétienne, en assimilant systématiquement les théologiens chrétiens à des poètes ! Toute cette réflexion sur la poésie s'inscrit en fait dans une tactique anti-monothéiste.

\section{Détordre la poésie: le cours sur Homère et son Iliade}

Pléthon condamne donc clairement la poésie, au profit des doctrines philosophiques originelles, transmises en vers et restaurées. Pourtant, selon lui, la poésie conserve des traces, des vestiges de la vérité philosophique et si l'on recueille ces traces, on peut „détordre“ ce que les poètes ont tordu $^{36}$ : on peut remonter de ce qui est corrompu à une doctrine saine.

C'est ce qui se passe avec un vers orphique que Pléthon considère comme juste d'un point de vue philosophique, et cite à deux reprises dans la Réplique à Scholarios ${ }^{37}$ : „car dans l'opinion

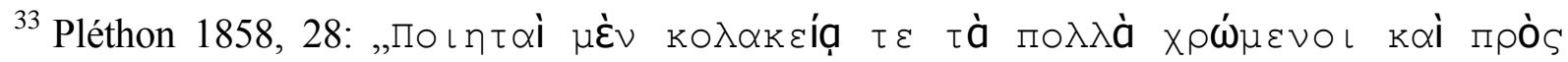

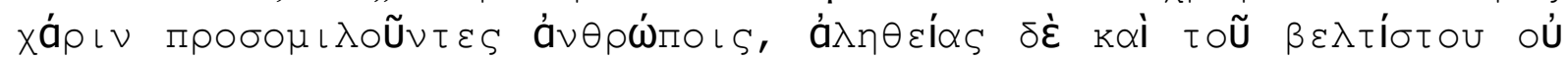

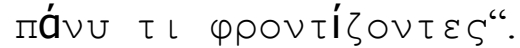

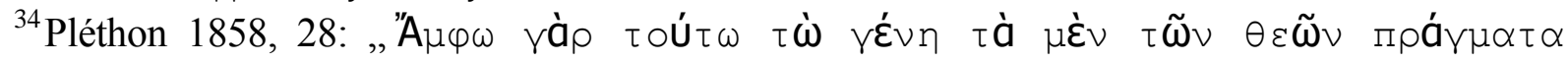

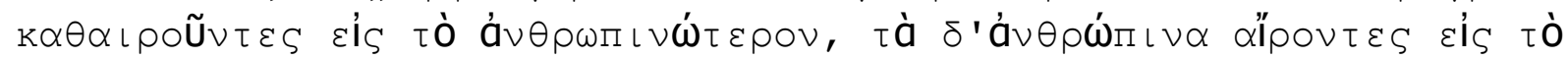

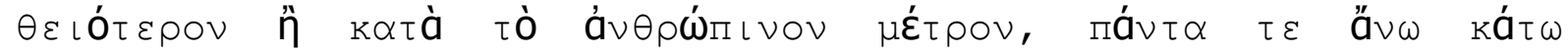

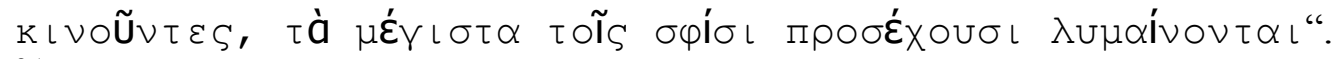

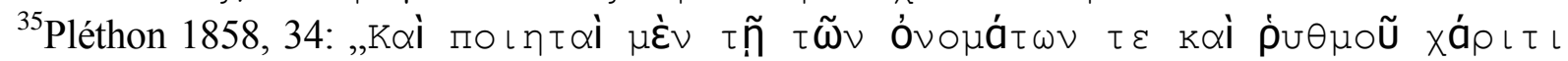

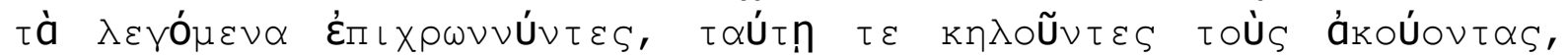

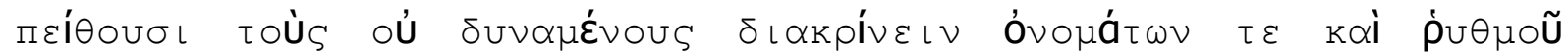

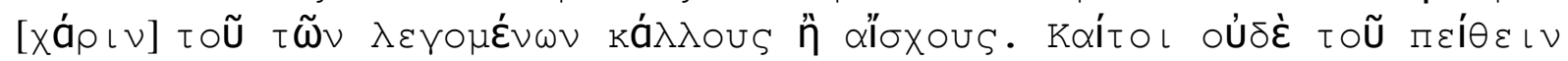

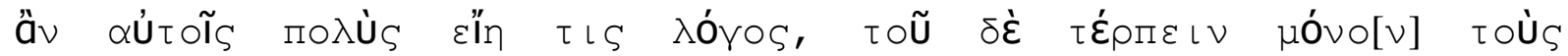

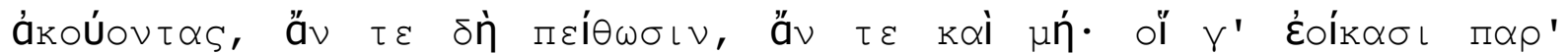

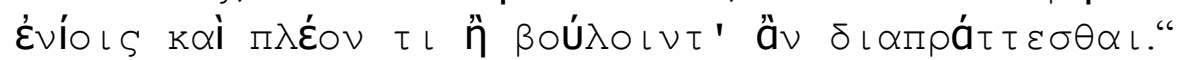

${ }^{36}$ Voir ci-dessus, note 29.

${ }^{37}$ Rappelons que pour Diogène Laërce (Vitae philosophorum, Prooemium, 5), ceux qui veulent attribuer aux étrangers l'invention de la philosophie, présentent Orphée comme le plus ancien philosophe.
} 
commune des Grecs dominait surtout cette croyance que 'de Zeus toutes choses ont surgi'، ${ }^{38}$; ,,et aussi ce que je cite d'Orphée, bien antérieur [à Aristide Aelius]: 'de Zeus, dit-il, toutes choses ont surgi'“39. Pléthon a d'ailleurs lui-même copié dans ses cahiers personnels conservés dans le manuscrit grec 406 de la Biblioteca Marciana, des hymnes orphiques et une «Théologie orphique sur Zeus » extraite du De Mundo attribué à Aristote; ils accompagnent son Traité de musique $e^{40}$.

Mais dans la Réplique à Scholarios, Pléthon prend soin aussitôt de rappeler qu'il faut user de prudence avec les poètes ${ }^{41}$ : „Les Grecs avaient de l'indulgence pour les poètes, lorsque leurs chants comportaient quelque chose de choquant envers les dieux (ce qui était fréquent), car les poètes glissent dans les fictions ${ }^{42}$; mais contre les sages qui se permettaient le même écart, ils s'irritaient fortement. Anaxagore courut le danger d'être accusé pour impiété، “43.

C'est sans doute dans le petit traité inédit de Pléthon Sur Homère et son Iliade ${ }^{44}$ que l'on trouve le meilleur exemple de „redressement“ philosophique d'une histoire transmise par un poète. Cet opuscule comporte tout d'abord une ,Vie d'Homère“ qui servait manifestement d'introduction à un cours que Pléthon dispensait ${ }^{45}$. Elle est suivie d'une «Division des dieux »: il s'agit d'une classification dans laquelle Pléthon tantôt justifie, tantôt corrige, les fonctions des dieux à l'égard des Grecs et des Troyens, d'un point de vue philosophique. Les règles de son interprétation se trouvent dans le Traité des lois $^{46}$. Ainsi, Poséidon, le dieu «le plus puissant après Zeus », dieu de la forme et de la limite (peras) dans le Traité des lois, impose des limites à la guerre entre les Grecs et les Troyens. Héra, déesse de la matière présidant à la multiplication des êtres, épouse de Poséidon (non de Zeus) dans le Traité des lois, veille sur les lois du mariage et tend, dans L'Iliade, à les faire respecter. Héphaïstos, défini comme le dieu de l'immobilité et du repos (stasis) dans le Traité des lois, veille sur ce qui est établi: il pousse à observer les lois du mariage et celle de l'hospitalité pendant la guerre de Troie; il apporte son secours, lorsque les lois sont transgressées, en vue de leur rétablissement. Dans le Traité des lois, Athéna est préposée à l'hétéro-mouvement (opposé à l'auto-mouvemement). Elle meut vers autrui, et pousse les Grecs à attaquer les Troyens. Hermès, dans le Traité des lois, régit les démons qui

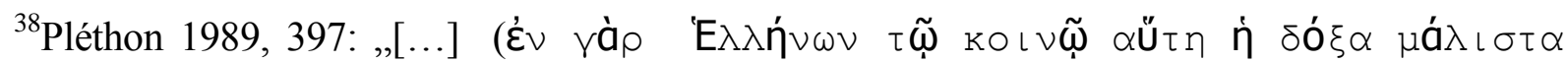

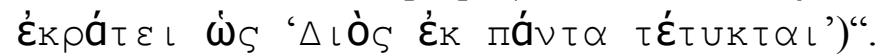

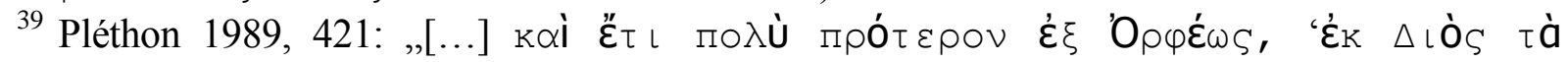

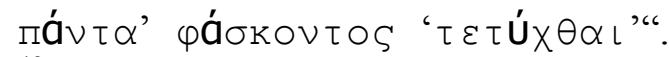

${ }^{40}$ Voir la description de ce manuscrit dans Mioni 1985, 159.

${ }^{41}$ Voir Pléthon 1989, 421 et Pléthon 1858, 29, 35 et surtout 131.

${ }^{42}$ Pléthon 1989, 421. Le terme de huponoia est employé par Platon, République, II, 378 d 6.

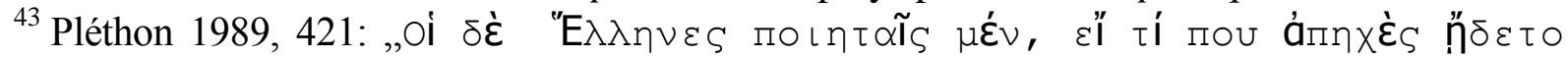

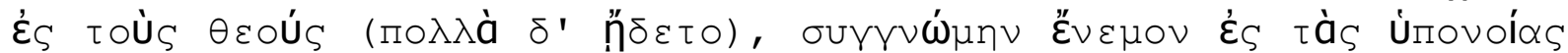

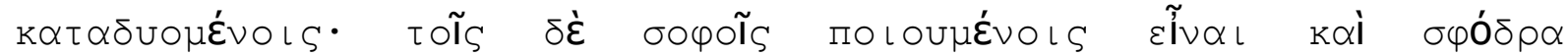

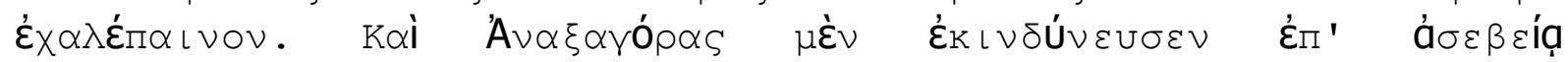

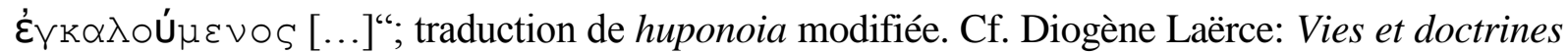
des philosophes illustres, II, 12-13; art. „Anaxagoras“ (n¹981; Harpocration et Hésychius), in: Suidas ${ }^{2} 1971,178,1-14$.

${ }^{44}$ Voir ci-dessus, note 26.

${ }^{45}$ On sait que Pléthon avant d'être envoyé à Mistra enseignait à Constantinople et qu'il a eu par exemple pour élève Marc Eugénikos, le futur Marc d'Ephèse: voir les références dans Tambrun 2006, 37-38. Sur le genre littéraire des „Vies d'Homère“, voir par exemple Homeric Hymns 2003.

${ }^{46}$ Tambrun 2006, 145-172.
} 
sont des messagers. Pendant la guerre de Troie il s'occupe donc des ambassades, règle les demandes et les échanges. Il pousse les Grecs à réclamer Hélène et les biens dérobés. Apollon et Artémis sont dans le cours Sur Homère et son Iliade, comme dans le Traité des lois, respectivement préposés à l'identité (tautotês) et à l'altérité (heterotês). Apollon favorise la concorde entre les peuples divers, tandis qu'Artémis pousse à leur distinction, à leur séparation, ce qui est facteur de dissension. Léto se trouve dans le Traité des lois, préposée à l'éther qui est la partie la plus brillante de l'élément du feu ${ }^{47}$. C'est donc elle - et non Zeus - qui aide les Troyens à mettre le feu aux navires des Grecs ${ }^{48}$ pour les détruire. Dans le Traité des lois, Aphrodite fait partie de la famille bâtarde - et non de la famille légitime - des dieux issus de Zeus; cette famille régit le domaine du vivant mortel. Aphrodite est préposée à la matière des vivants mortels. Dans L'Iliade elle procure le plaisir et favorise ainsi la relation entre Hélène et Alexandre, ce qui déclenche la guerre de Troie. Arès est absent du panthéon du Traité des lois ${ }^{49}$. Selon l'empereur Julien, il ne peut s'agir que d'un dieu ethnarque. De même, selon Pléthon, Arès ne possède, lors de la guerre de Troie, qu'un rôle particulier: celui de protecteur de la ville de Troie. Pléthon fait remarquer qu'Héphaïstos est au contraire un dieu protecteur général qui rétablit les lois pour tous.

Le cours Sur Homère et son Iliade se termine par une mise en garde du professeur Pléthon: il a «redressé » L'Iliade d'Homère en supprimant les mythes franchement absurdes; mais pour ce qui reste encore dans ce texte et qui, sans être aussi absurde, n'est pourtant pas correct, il ne faut pas tout étudier à la suite avec précipitation; mais c'est en analysant chacune des choses de ce genre au moyen de la droite raison, qu'il faut aussi y prêter l'oreille, attendu qu'il n'est pas facile de les supprimer. Ainsi, pour le reste du texte, Pléthon de mande de faire preuve d'esprit critique !

\section{Remplacer les mythes poétiques par des mythes corrects}

Ce n'est pas parce qu'elle fournit des mythes, que Pléthon condamne la poésie. Suivant Platon (République II, 377 b), il estime en effet que la forme mythique peut avoir une utilité pédagogique pour tous ceux qui ne sont pas capables de spéculations philosophiques difficiles, et donc notamment pour le peuple. Il préconise de remplacer les mythes déformés des poètes, par des mythes philosophiques. Dans sa Défense d'Aristote (Contre les difficultés de Pléthon au sujet d'Aristote $)^{50}$, Georges Scholarios soutient que „l'obscurité d'Aristote convient à un sage, le langage coloré de Platon davantage à un poète, pour ne pas dire à un genre encore inférieur's51. Pléthon rétorque alors: „La forme mythique peut avoir quelque fondement. Si les mythes, en effet, n'étaient remplis de bavardage dans la mesure où ils viennent des poètes, ils fournissent à la foule, qui ne peut atteindre la profondeur de la pensée, une compréhension qui soit à sa portée ${ }^{52}$; c'est ce à quoi Platon aussi s'appliqua, par philanthropie, à la suite d'autres théologiens. Détournant des mythes pervers des poètes la foule éprise de mythes, lui-même, par d'autres mythes bienfaisants ${ }^{53}$, donna à cette foule aussi de comprendre quelque chose du divin, qui ne

\footnotetext{
${ }^{47}$ Voir Tambrun 2006, 150.

${ }^{48}$ Comparer avec L'Iliade, XVI.

${ }^{49}$ Voir Tambrun 2006, 148.

${ }^{50}$ Dans Scholarios 1928-1936, IV, 1-116.

${ }^{51}$ Texte cité par Pléthon dans sa Réplique à Scholarios: Pléthon 1989, 382.

${ }^{52}$ Voir Grégoras 1975, 965-967.

${ }^{53}$ Comparer notamment avec les Prolégomènes à la philosophie de Platon, 7, 24-8, 2 (Prolégomènes 1990, 11-12); Proclus 1899 (In Rempublicam, VI ${ }^{\mathrm{e}}$ dissert.), I, 71-86; 159-163;
} 
lui fût pas trop étranger, afin que les sages et la foule puissent ensemble y accéder quelque peu ${ }^{54}$. De l'obscurité du style, les sages retireront un effort supplémentaire, là où il était possible de penser avec moins de peine, la foule, elle, n'en recueillera pas le moindre avantage ${ }^{\text {c55 }}$. C'est ainsi que Pléthon restaure et transforme, par exemple, le mythe des Courètes ${ }^{56}$, des Géants ${ }^{57}$, ou encore d'Héraclès, des Héraclides, ou de Dionysos ${ }^{58}$.

\section{Conserver les noms divins même souillés par les poètes}

Dans la préface du Traité des lois, Pléthon indique que „Cet ouvrage contient la théologie d'après Zoroastre et aussi d'après Platon, en désignant les dieux reconnus par la philosophie par des noms traditionnels propres aux Grecs, et en ramenant chacun d'eux, du sens moins conforme à la philosophie, pris à cause des distorsions $\left(\delta\left\llcorner\alpha \sigma \tau \rho \circ \varphi a_{S}\right)\right.$ accomplies par les poètes, au sens qui est le plus conforme à la philosophie ${ }^{659}$.

Dans un chapitre conservé „Sur les noms des dieux“600, il justifie à nouveau l'utilisation des noms traditionnels grecs des dieux ${ }^{61}$ : ils ont été transmis par les philosophes, mais détournés par les poètes. On peut néanmoins les utiliser après les avoir débarrassés des souillures ajoutées par les poètes. Ainsi, il s'agit de procéder à un nettoyage, comme pour les Oracles chaldaïques: „Cependant, pourrait-on dire, ces noms ont été complètement salis par les poètes qui ont fabriqué les mythes discordants à partir des discours philosophiques sur les dieux, et il ne fallait donc plus les utiliser. Mais en réalité, la nature des noms des dieux n'est pas telle que

Proclus 1968 (Théologie platonicienne, I, 4), 21, 1-22, 7; Ammonius 1895, 249, 1-25; Philopon 1897, 69, 30-70, 2; 116, 23-26; Olympiodore 1970, 237, 24-239, 30; 242, 18-243, 15.

${ }^{54}$ Voir Couloubaritsis 1997, 135-140.

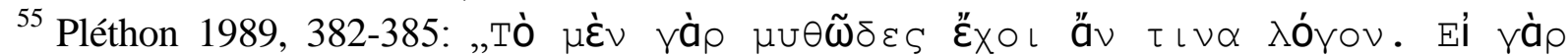

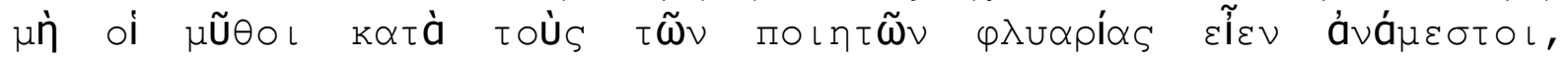

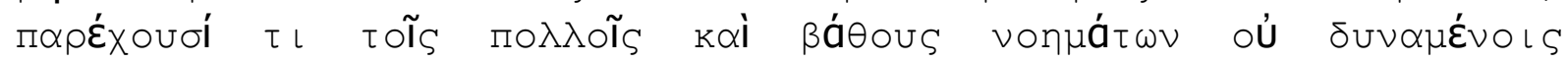

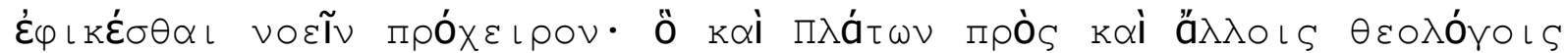

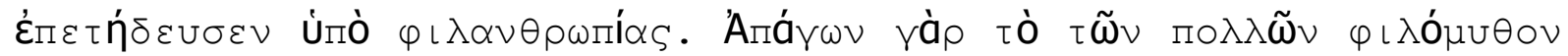

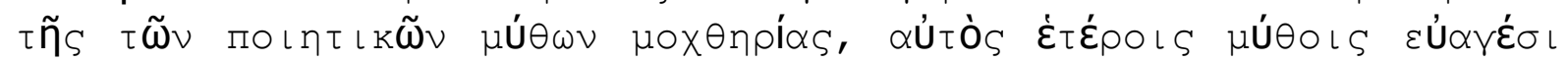

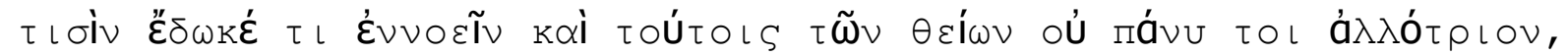

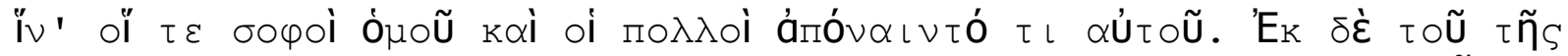

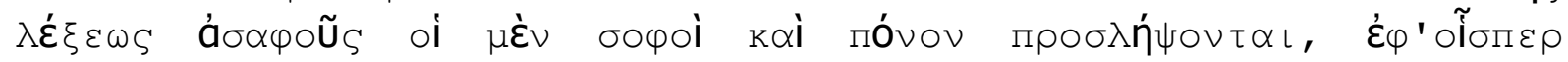

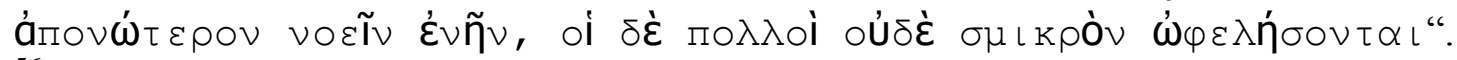

${ }^{56}$ Sur l'utilisation du mythe des Courètes chez Pléthon, voir Tambrun 2006, 86-87; 109; 149; 188.

${ }^{57}$ Sur l'utilisation du mythe des Géants chez Pléthon, voir Tambrun 2006, 87-88.

${ }^{58}$ Pléthon 1858, 30-32 et 252-256. Sur Héraclès, les Héraclides et Dionysos, on peut se reporter à l'index dans Tambrun 2006, 294-295.

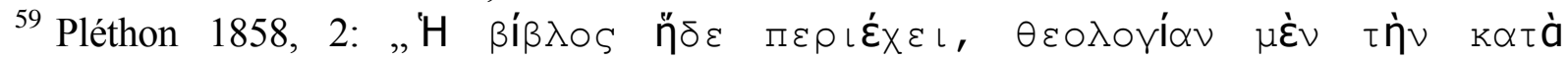

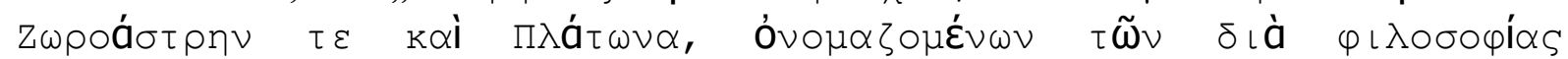

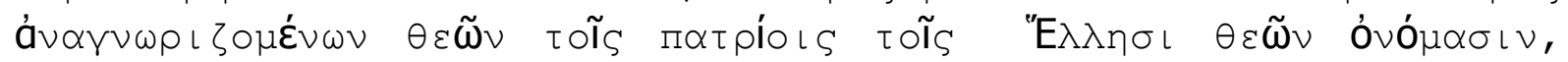

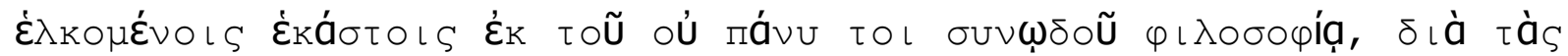

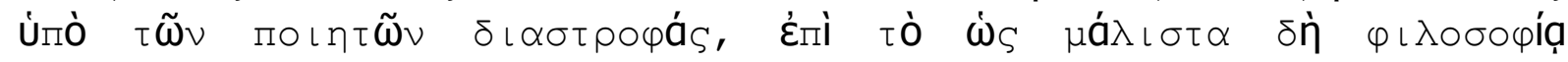

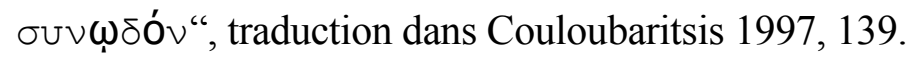

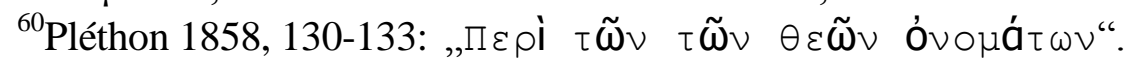

${ }^{61}$ Sur les noms et les attributions des dieux dans le panthéon de Pléthon, voir Tambrun 2006, 146-153. 
même si un nom est complètement sali, il le demeure à jamais [...], mais si quelqu'un d'autre emploie le même nom dans une acception saine et sainte, le nom lui aussi devient alors pur pour lui“62. En réalité, il ne s'agit pas tant de corriger les noms divins, que les attributs des dieux.

Mais la correction s'applique aussi à des textes philosophiques : en effet, il faut ajouter que Pléthon se permet tout de même - et sans le signaler - de corriger dans son édition des dialogues de Platon, tout ce qui lui paraît incorrect d'un point de vue théologique ${ }^{63}$. (On peut alors se demander s'il considère qu'il restaure les dialogues authentiques de Platon déformés au cours de leur transmission, ou bien s'il procède à une censure à l'encontre de Platon lui-même.)

En fait, si les noms des dieux corrompus par les poètes grecs (et parfois par les philosophes!) méritent d'être restaurés, c'est parce qu'ils témoignent d'un lien des Grecs avec le sol de l'Hellade. Le projet sous-jacent est politique: il faut rappeler aux Grecs qu'ils sont „Hellènes de

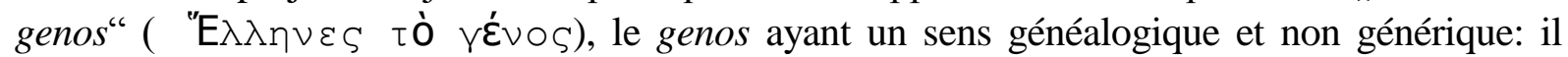
associe la lignée au sol, c'est à dire à la patrie ${ }^{64}$. Les noms des dieux sont des noms patriotiques ${ }^{65}$.

\section{Emploi pédagogique de citations de textes en vers}

Pour terminer sur ce point, il faut faire mention de deux citations de textes en vers, qui se trouvent dans la Réplique à Scholarios. Elles sont toutes les deux employées dans un but pédagogique: la courte citation d'un texte en vers est ici utilisée comme une maxime ou un

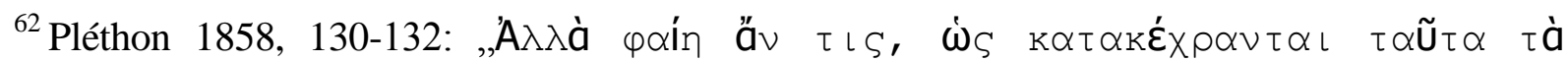

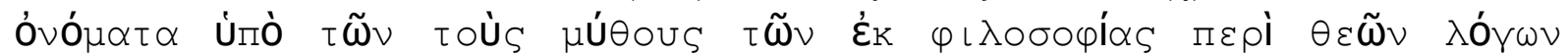

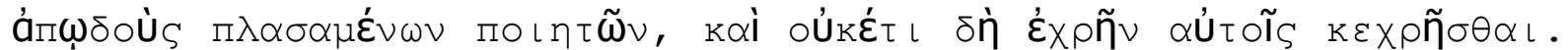

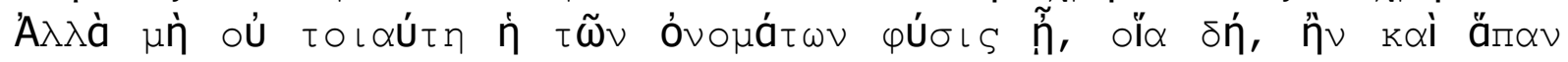

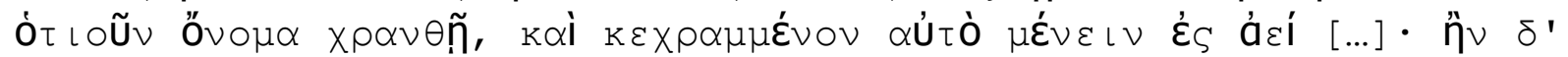

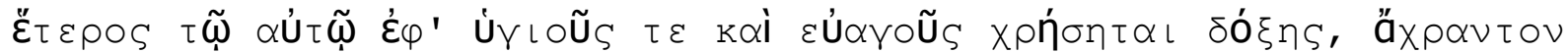

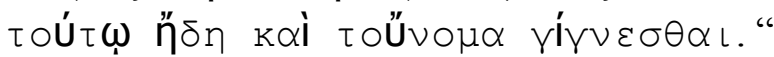

${ }^{63}$ Voir Pagani 2009.

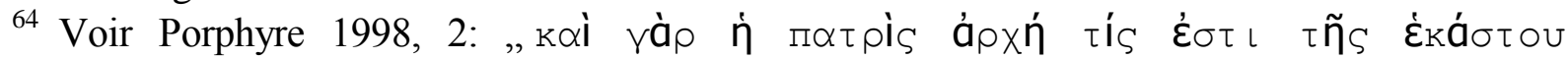

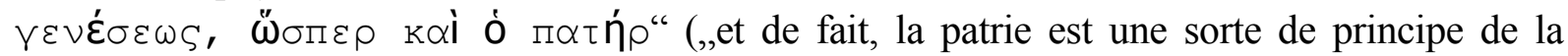
naissance de chacun, tout comme le père"). Sur la différence entre le genos au sens générique et au sens généalogique, voir Tambrun 2006, 175-185.

65 Ainsi, Pléthon écrit à l'empereur Manuel II Paléologue (Pléthon 1926, 247, 14-248, $5)$, ,[...] nous, que vous gouvernez et dont vous êtes l'empereur nous sommes Hellènes de genos, comme l'attestent notre langue et la culture de nos pères. Et pour les Grecs il n'est pas possible de trouver un pays qui leur soit plus propre et qui leur convienne mieux que le Péloponnèse et toute la partie de l'Europe qui lui est contiguë ainsi que les îles adjacentes. En effet, c'est manifestement le pays que les Grecs eux-mêmes ont toujours habité, du moins d'après les souvenirs que les hommes ont conservés; personne d'autre ne l'avait habité avant eux

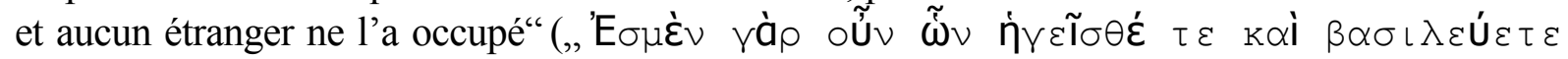

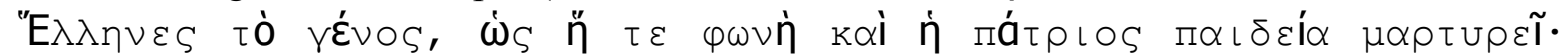

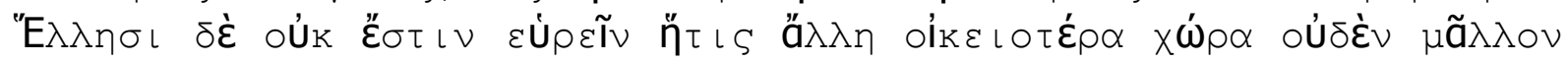

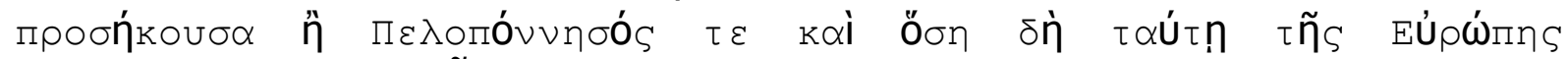

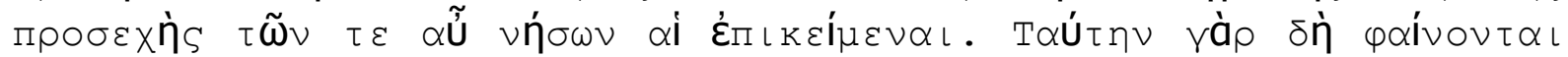

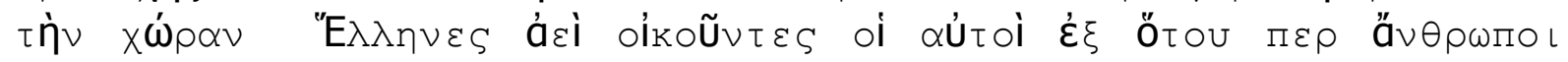

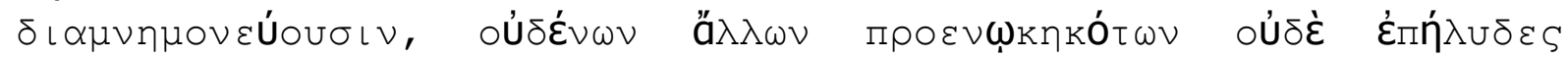

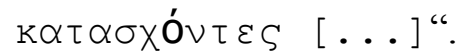


aphorisme qui fait autorité: on doit suivre le mot d'ordre ou l'exemple, lorsque l'on n'est pas encore capable de raisonner philosophiquement et que l'on s'entête dans des préjugés. Cet usage pédagogique de la citation, qui nous montre l'un des aspects de l'enseignement littéraire à Byzance, est d'ailleurs déjà recommandé par Plutarque dans le cadre d'un enseignement préparatoire à la philosophie ${ }^{66}$. Dans la Réplique à Scholarios, la première citation est constituée de trois Vers d'or pythagoriciens ${ }^{67}$. La seconde, à la fin de l'ouvrage, est une citation d'Euripide $^{68}$ : il s'agit de montrer que l'homme qui se plait en mauvaise compagnie est semblable à ceux qu'il fréquente ${ }^{69}$; Pléthon veut faire comprendre à Scholarios qui s'entête dans l'aristotélisme et le thomisme, qu'en compagnie d'Aristote, il se trouve en fort mauvaise compagnie. C'est le coup de bâton final d'un vieux maître agacé, à l'arrogant borné qui aurait pu être son élève !

\section{Composition d'Hymnes aux dieux}

Pléthon compose pour sa part des Hymnes aux dieux strictement philosophiques. Ils font concurrence à ceux de Proclus que Pléthon a copiés dans ses cahiers personnels ${ }^{70}$. Ils sont au nombre de 27 (soit $3 \times 3 \times 3$ ). Chacun comporte neuf vers, que l'on doit scander ,dans le ton de l'hexamètre“" ${ }^{71}$, le mètre héroïque étant „le plus beau des rythmes“"72. La justification est rythmique: le mètre héroïque ne comporte que deux pieds: le dactyle (une longue pour le temps frappé, deux brèves pour le temps levé) et le spondée (une longue pour le frappé, une longue pour le levé): „Ainsi ces deux pieds commençant tous deux par une longue et se terminant au

${ }^{66}$ Voir par exemple Brechet 2005.

${ }^{67}$ Pléthon 1989, 422-423; B. Lagarde (Pléthon 1989) cite les Vers d'or (vers 40-42) attribués à Pythagore, dans l'édition d'A. Farina (Pythagore 1962, 23):

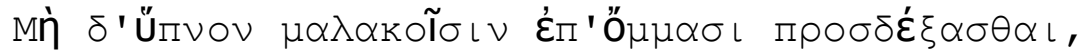

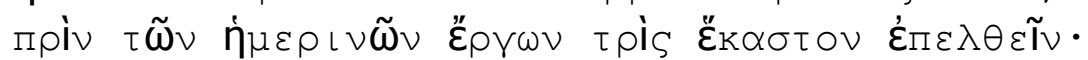

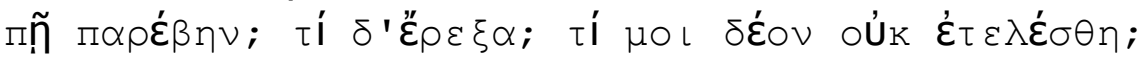

$$
\begin{aligned}
& \text { N'accueillez pas le sommeil sur vos yeux assoupis, } \\
& \text { avant d'avoir parcouru trois fois chacune des actions de la journée. }
\end{aligned}
$$

En quoi ai-je transgressé ? Qu'ai-je fait ? Que n'ai-je pas accompli ce qui devait l'être ?

Par ailleurs, signalons que Pléthon a copié des Vers d'or dans ses cahiers personnels, conservés dans le Marcianus graecus 406, aux folios 121v-122v: voir Mioni 1985, 159.

${ }^{68}$ Pléthon 1989, 498-499; Euripide, Phoenix 1964, 623 (fr. 812, 7-9):

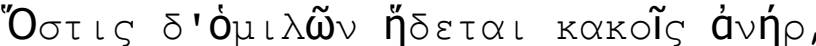

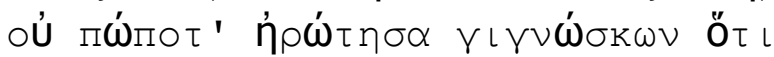

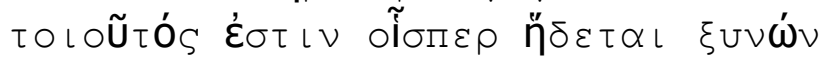

Un homme qui se plaît en mauvaise compagnie,

Je ne me suis jamais enquis de lui, sachant qu'il est tel que ceux qu'il se plaît à fréquenter

${ }^{69}$ Pléthon 1989, 499, n. 306.

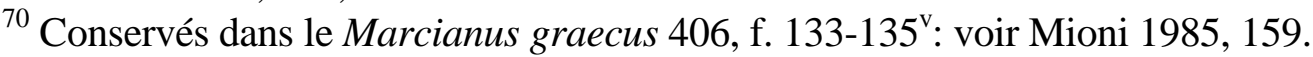

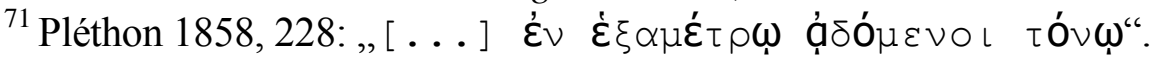

72 Pléthon 1858, 228; cf. par exemple, Aristide Quintilien 1963, 47 (Aristide Quintilien 1999, 106). 
temps levé, étant de plus égaux pour la mesure, donnent à ce rythme un caractère de noblesse dont nul autre n'approche ${ }^{673}$. Le temps fort se situe donc sur la première syllabe de chaque mètre. A la suite de ces Hymnes en vers, Pléthon donne dans le Traité des lois, des „Instructions pour l'usage des allocutions et des hymnes“674 (les „Allocutions aux dieux“ ne sont pas en vers). Il donne pour l'année entière un emploi du temps extrêmement précis et très contraignant qui varie

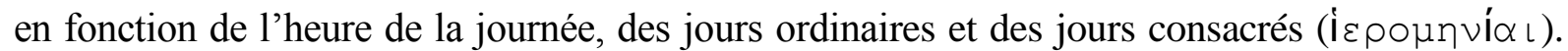
De ce long exposé très détaillé, nous retiendrons que les Hymnes sont tantôt seulement scandés, tantôt chantés. Lorsqu'ils sont chantés, ils le sont selon les harmonies musicales définies par Platon dans la République (III, 398-399). Ces „harmonies“ ont comme chez Platon un caractère éthique: elles sont propres à favoriser un comportement vertueux. Il faut comprendre qu'en accordant les parties de l'âme comme les cordes d'une lyre, elles accordent la cité humaine au monde et à la communauté des dieux. Il est clair pour Pléthon qu'elles n'ont aucune efficacité sur les dieux qui sont parfaitement immuables: elles nous accordent aux dieux.

Le traité de musique de Pléthon intitulé Sur quelques points des rapports musicaux, se trouve à la fin de l'édition du Traité des lois ${ }^{75}$. C'est ce texte qui a d'ailleurs donné à A. J.-H. Vincent qui menait des recherches sur la musique grecque antique, l'idée de s'intéresser aux fragments du Traité des lois présents dans les mêmes manuscrits ${ }^{76}$. Il comporte classiquement des éléments d'harmonique (commençant par la phonologie), de rythmique et de métrique. Il s'agit comme le petit traité Sur Homère et son Illiade, d'un ouvrage de Pléthon destiné à l'enseignement ${ }^{77}$.

Pour l'harmonique, Pléthon définit les intervalles selon une approche strictement pythagoricienne, par des rapports mathématiques de proportion se rapportant à la longueur des cordes $^{78}$. Rappelons qu'un rapport harmonique est un rapport superpartiel (ou épimore): le numérateur y est supérieur d'une unité au dénominateur: $(n+1) / n$. L'intervalle fondamental

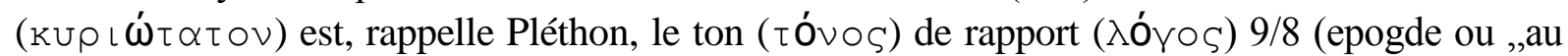
dessus du huit"). Pléthon définit ensuite dans l'ordre: le système épitrite 4/3 (la quarte), composé de deux tons et d'un intervalle inférieur à un demi-ton - ce qui montre à nouveau que Pléthon

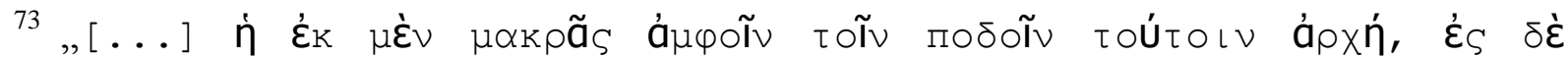

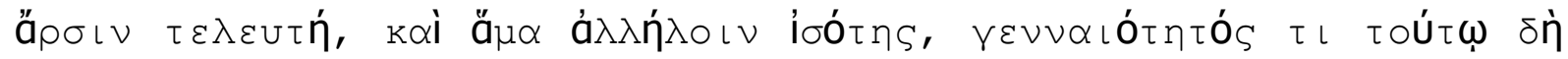

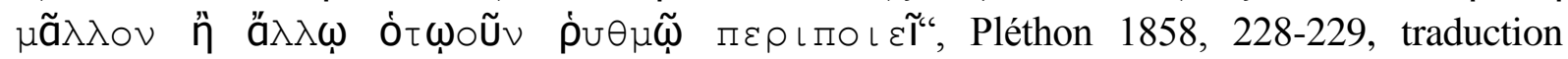
[Pellissier] légèrement modifiée. Sur la vertu de „noblesse“ ( $\vee \varepsilon \vee \vee \alpha \iota$ ó ๆ $\varsigma$ ) qui est l'une des parties du courage, voir le traité Des vertus: Pléthon 1987, 4, 7, 15, 21, 23, 28. Pléthon semble ici suivre l'Essai sur la vie et la poésie d'Homère (VII) du Pseudo-Plutarque.

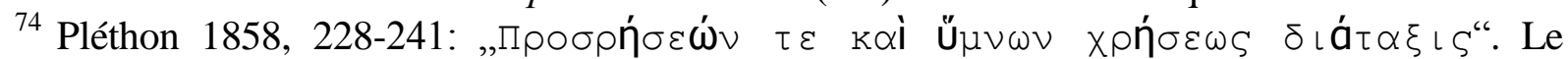
chapitre ne nous est pas parvenu en entier.

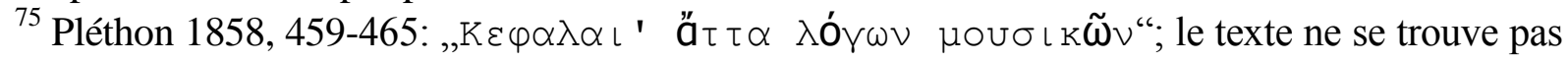
dans la réimpression de 1982 (Paris: Vrin).

${ }^{76}$ Voir la notice préliminaire au Traité des lois (Pléthon 1858, I-IV).

${ }^{77}$ Sur l'enseignement universitaire à Byzance voir en général Fuchs 1926; pour les XIII ${ }^{\mathrm{e}}$ et XIV ${ }^{\mathrm{e}}$ siècles, voir la préface de Vitalien Laurent à l'édition du Quadrivium de Georges Pachymère (Pachymère 1940, XVII-XXXIII); pour le XV siècle, voir notamment Cacouros 1996 et Cacouros 1997.

${ }^{78}$ Sur les deux principaux courants, pythagoricien (s'intéressant exclusivement aux rapports mathématiques) et aristoxénien (privilégiant l'approche par l'écoute), et la tentative de conciliation effectuée par Claude Ptolémée, voir la synthèse de Bélis 1996. Les traités de musique les plus importants dans la dernière période de Byzance sont ceux de Georges Pachymère (deuxième partie du Quadrivium, voir ci-dessus, note 77) et de Manuel Bryennios (Bryennios 1970); voir aussi Métochite 2007. 
s'inscrit dans le courant pythagoricien et non aristoxénien. Il définit le plus petit intervalle

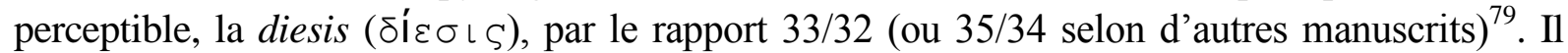

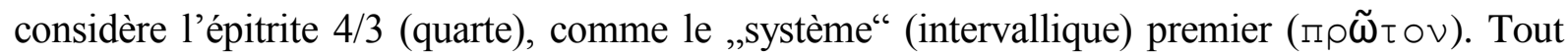
système (intervallique) se résout en épitrite 4/3 (quarte) et hémiole 3/2 (quinte), l'hémiole surpassant l'épitrite d'un ton. Pour la composition de l'octave qui est, explique Pléthon, de six tons, ou mieux de cinq tons et deux demi-tons, et de rapport 2, il existe deux possibilités: soit l'épitrite et l'hémiole (quarte et quinte), soit deux épitrites et un ton (deux quartes et un ton). Le ton est classiquement considéré comme la différence entre la quarte et la quinte; il est donc, conformément à l'enseignement des pythagoriciens, le pivot central du système que constitue l'octave composé de deux quartes et d'un ton central ${ }^{80}$. Notons ici que les intervalles définis par ces rapports mathématiques, ne sont pas ceux du tempérament égalisé du piano: la quarte juste 4/3 dont parle Pléthon est un peu plus basse que celle de nos instruments modernes, et la quinte juste $3 / 2$, un peu plus haute; le ton $9 / 8$ est plus haut également ${ }^{81}$.

Malheureusement, Pléthon ne donne pas de précisions techniques sur les „harmonies“ qu'il préconise pour leur caractère éthique (hypodorienne et dorienne, hypophrygienne et phrygienne) et dans lesquelles il demande de chanter les Hymnes aux dieux. Comme Platon, il emploie des adverbes signifiant „,en dorien“( $\delta \omega \rho\llcorner\sigma \tau i ́)$, ,en phrygien“( $\varphi \rho v \curlyvee\llcorner\sigma \tau i ́)$, etc., et non des noms en - o $\vee$ ou des adjectifs en -oS, d'un usage plus tardif; de plus, il emploie le terme d',harmonie“ (áphoví $\alpha$ ) utilisé à l'époque de Platon ou par les auteurs archaïsants, et non celui de „ton“ (tonos), d',,aspect [d'octave]“ (eidos) ou encore de „lieu de la voix“ (topos phônês), c'est-à-dire de clef, utilisés par la suite. On peut donc se demander si Pléthon cherche simplement à employer un vocabulaire de type platonicien, ou bien s'il parle véritablement des „harmonies“" telles qu'elles étaient définies à l'époque de Platon.

On se souvient que de toutes les harmonies antiques, Platon n'en retient que deux qui seraient propres à conduire les gardiens de la cité, respectivement au courage dans les combats, et à la sagesse en temps de paix: l'harmonie dorienne et la phrygienne. Par le pythagoricien Aristide Quintilien qui a vécu entre le $\mathrm{I}^{\mathrm{er}}$ et le $\mathrm{III}^{\mathrm{e}}$ siècle de notre ère, et qui commente le fameux passage de la République (III, 398-399) $)^{82}$, nous connaissons ces modes très anciens et leur structure ${ }^{83}$. Le dorien des „très anciens“ possède la structure suivante (du grave à l'aigu): ton-diésis-diésisditon-ton-diésis-diésis-diton (dépassant ainsi d'un ton l'intervalle d'octave); leur phrygien possède la structure suivante: ton-diésis-diésis-diton-ton-diésis-diésis-ton (octave parfaite). Mais Pléthon parle de deux harmonies supplémentaires qui ne se trouvent pas chez Platon: l'hypodorien et l'hypophrygien, ainsi que de leur ethos. On suppose que les deux modes nommés à la fin du $\mathrm{V}^{\mathrm{e}}$ siècle ou au début du $\mathrm{IV}^{\mathrm{e}}$, „hypodorien“ et „hypophrygien“, étaient obtenus par des modulations introduites dans le dorien et le phrygien, en remplaçant les tétracordes disjoints par des tétracordes conjoints; mais leur structure ne nous est pas connue avec précision ${ }^{84}$. Comme Aristide Quintilien ne parle pas de ces deux modulations, Pléthon doit plutôt se référer aux dorien, phrygien, hypophrygien et hypodorien, tels qu'ils ont été définis ultérieurement par Aristoxène de Tarente, par divers théoriciens de l'époque hellénistique et par Claude Ptolémée. Ils sont alors considérés comme des ,aspects d'octave“ de forme régulière et

\footnotetext{
${ }^{79}$ Voir Pléthon $1858,465-466$ (note f).

${ }^{80}$ Sur l'origine de ce système musical, voir Wersinger 2008, 130-131 et 294-296.

${ }^{81}$ Je renvoie à Reznikoff 1981 et Reznikoff 1988: ces deux articles sont en ligne sur le site Internet de l'association Ecoledelouange.

${ }^{82}$ Voir aussi Platon, Lachès 188d, 193d.

${ }^{83}$ Le texte se trouve, avec la structure des échelles dans Aristide Quintilien 1963, 18-19 (Aristide Quintilien 1999), 51-54. Voir aussi West 1992, 174-175.

${ }^{84}$ Voir West 1992, 183.
} 
compatibles avec le ,système parfait“, mais en fait dénaturés ${ }^{85}$, surtout lorsqu'ils en viennent à être employés simplement comme „tons“ ou comme clefs (topoi phônês) permettant les transpositions - et c'est encore en ce sens qu'ils sont employés dans les traités de musique de l'époque byzantine. Aristide Quintilien explique comment ils sont engendrés ${ }^{86}$. Dans le genre diatonique, le dorien est constitué de deux tétracordes doriens („,demi-ton“-ton-ton) ${ }^{87}$ séparés par un ton entier disjonctif, et l'hypodorien est constitué de deux tétracordes doriens conjoints, et d'un ton entier au grave; de même, le phrygien est constitué de deux tétracordes phrygiens (ton„demi-ton“-ton) séparés par un ton entier disjonctif, et l'hypophrygien est constitué de deux tétracordes phrygiens conjoints, et d'un ton entier au grave.

S'appuyant sur cette conception de l',harmonie“ en fait définie comme ,aspect d'octave“, Pléthon propose son propre système de valeurs éthiques ${ }^{88}$. Ainsi, on chante, explique-t-il, en

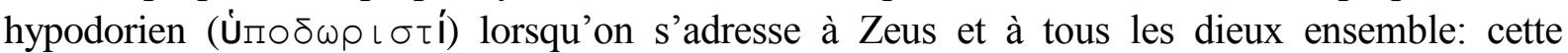

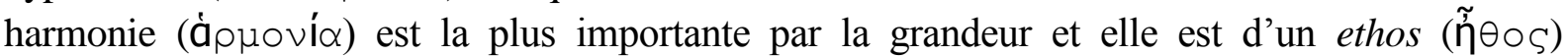

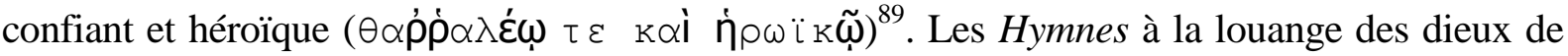

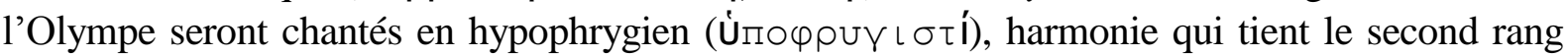
pour la grandeur et dont l'ethos convient à l'admiration des belles choses. Les Hymnes aux dieux

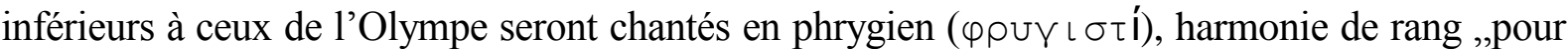

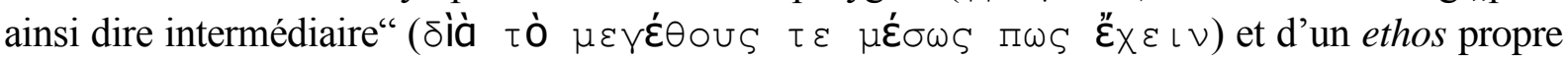

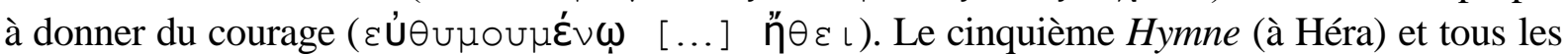
Hymnes quotidiens se chanteront en dorien ( $\delta \omega \rho\llcorner\sigma \tau i ́)$, harmonie (áa $\rho \circ v i ́ \alpha)$ qui est assignée aux hommes, et à la divinité qui préside aux destinées humaines; elle est d'un ethos

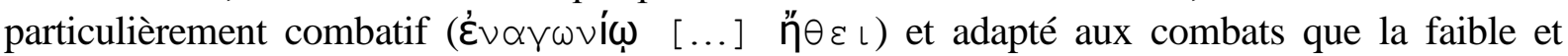
fragile nature humaine doit toujours livrer ${ }^{90}$.

Pléthon définit donc l'ethos des harmonies en fonction du panthéon qu'il établit dans le Traité des lois, tout en conservant au dorien (harmonie présidant à l'humain), l'ethos reconnu par Platon; le dorien présente d'ailleurs un caractère patriotique: rappelons que Platon le considère dans le Lachès (188d cf. Lettre VII, 336c), comme l'harmonie typiquement grecque! Le phrygien est bien une harmonie „intermédiaire“, mais traditionnellement plutôt parmi les trois harmonies primitives, c'est-à-dire entre le dorien (grave) et le lydien (aigu) ${ }^{91}$. Pour des raisons théologiques, chez Pléthon, l'hypodorien et le dorien encadrent, d'un point de vue éthique, l'hypophrygien et le phrygien qui forment un groupe intermédiaire entre les deux extrêmes. Proclus $^{92}$ dans la Chrestomathie groupait lui aussi l'hypophrygien et le phrygien pour en faire des harmonies propres au dithyrambe chanté en l'honneur du dieu Dionysos et inspirant

\footnotetext{
${ }^{85}$ Comme le montre West 1992, 185: „It became difficult to speak of mode except in terms of key. But in being interpreted as a function key, the modes were de-naturated. They lost all idiosyncrasy".

${ }^{86}$ Aristide Quintilien 1963, 17 (Aristide Quintilien 1999, 44-46).

${ }^{87} \mathrm{Du}$ grave à l'aigu. Comme nous l'avons vu, pour Pléthon, comme pour les pythagoriciens, l'intervalle qui complète le diton pour former la quarte, est inférieur à la moitié d'un ton.

${ }^{88}$ Voir l'exposé comparatif de F. Duysinx dans Aristide Quintilien 1999, 46 (n. 2). Les grands traités de musique de l'époque byzantine, celui de Georges Pachymère (Quadrivium, $\mathrm{II}^{\mathrm{e}}$ partie), et celui de Manuel Bryennios (Bryennios 1970) ne parlent plus de l'ethos du dorien, du phrygien, etc. (car ce ne sont plus que des clefs), mais seulement de l'ethos des mélodies.

${ }^{89}$ Pléthon 1858, 234.

${ }^{90}$ Pléthon 1858, 234.

${ }^{91}$ Voir Ptolémée 1930, 62,19; Pachymère 1940, 198, 19; Bryennios 1970, 118, 23-120,1.

${ }^{92}$ Il s'agit probablement de Proclus de Sicca, le grammairien du II ${ }^{\mathrm{e}}$ siècle.
} 
l'enthousiasme divin ${ }^{93}$. Selon Pléthon, la première harmonie (l'hypodorien) est dédiée au dieu suprême, père de tous les dieux et (par différents intermédiaires) des hommes, la quatrième (le dorien), se rapporte à l'humain.

Les indications de Pléthon sur la métrique comme sur l'harmonique sont seulement élémentaires. En effet, s'il accorde une grande importance à toute la diversité de la paideia, donc au Trivium et au Quadrivium, il n'est pas question de perdre de vue le but qui est politique: il ne s'agit pas de former des musiciens ou des poètes professionnels, mais plutôt des citoyens vertueux et courageux.

Rappelons que les Byzantins ont redécouvert la métrique au début du XIV ${ }^{\mathrm{e}}$ siècle. C'est Démétrius Triclinius ${ }^{94}$ qui fut le premier Byzantin à comprendre l'intérêt de la métrique pour l'étude de la poésie ancienne ${ }^{95}$, et il l'appliqua aux œuvres théâtrales en corrigeant les vers iambiques (on lui doit la découverte d'un manuscrit contenant neuf tragédies d'Euripide qui était auparavant peu connu des Byzantins). On peut considérer que Pléthon continue le travail de correction métrique que Triclinius avait engagé pour le théâtre, en l'appliquant aux Oracles chaldaïques pour retrouver derrière cette collection les Oracles magiques des mages disciples de Zoroastre.

Pour conclure sur ce point, nous dirons que Pléthon refuse de voir dans la poésie une source fiable pour la théologie qu'il restaure. La poésie n'est qu'un divertissement. Néanmoins, comme Pléthon veut rappeler à ses contemporains leur lien au sol de l'Hellade, leur demandant de le défendre et de le reconquérir, il attache finalement de l'importance aux noms divins transmis en langue grecque par les poètes. Il conserve donc ces noms mais rectifie les attributs des dieux déformés par les poètes. Ceux-ci à partir d'une théologie originelle juste au départ, auraient forgé des fables dans le but de plaire à leur public; les „sophistes“ ou théologiens chrétiens leur sont associés. Néanmoins, dans un but pédagogique, lorsque l'on s'adresse à une foule non érudite, le genre du mythe ou de la fable peut être utilisé, mais il doit toujours s'agir comme chez Platon, de mythes philosophiques, non de mythes poétiques.

Il faut souligner que la dissociation entre poésie et versification est facilitée par le fait que la métrique fait partie des disciplines musicales, à l'instar de l'harmonique, et donc des disciplines

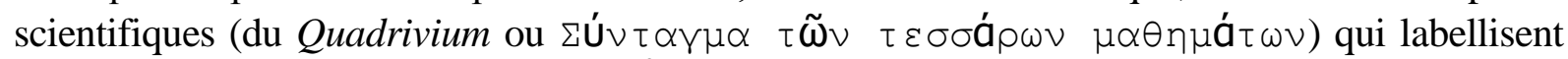
le philosophe ou le savant de bon aloi ${ }^{96}$. La poésie, quant à elle, ne relève que des disciplines littéraires (du Trivium).

Enfin, il est clair que si Pléthon attache tant d'importance à la musique qui comporte la métrique, la rythmique et l'harmonique, c'est parce qu'il se soucie de ses effets éthiques, c'est-à-dire des mouvements qu'elle produit sur le pneuma éthéré. Ce pneuma est le corps très subtil auquel l'âme est toujours unie; il est le siège des perceptions et de l'imagination, et il sert à l'âme de véhicule dans sa descente dans le corps mortel et lors de sa remontée vers sa patrie céleste. La musique doit disposer à la vertu et à la mise en harmonie de la société humaine avec la communauté de dieux.

\footnotetext{
${ }^{93}$ Tandis que le lydien était propre au nome, dédié au dieu Apollon et inspirant l'ordre et la magnificence. Mais le lydien est exclu par Platon pour l'éducation des gardiens; en bon platonicien, Pléthon ne peut donc pas l'utiliser. Le texte se trouve dans Photius 1967, 161 (cod. 239).

${ }^{94}$ Il enseigna à Thessalonique d'environ 1305 à 1320.

${ }^{95}$ Reynolds/Wilson ${ }^{3} 1991,51-52$.

${ }^{96}$ Voir la préface de V. Laurent, dans Pachymère 1940, XVIII.
} 


\section{Les réactions des contemporains de Pléthon}

Les réactions des contemporains de Pléthon sont très contrastées. Du côté des Grecs, quelques années après la mort de Pléthon, Georges Scholarios, devenu patriarche de Constantinople, brûle le manuscrit autographe de Pléthon, tout en conservant, comme preuve à charge, les Hymnes et les Oracles! Il interdit d'en prendre copie, ce qui a pour effet d'intriguer la cour ottomane qui fait traduire les restes du manuscrit en arabe ! C'est ainsi que possédons une traduction arabe des Oracles et du reste du Traité des lois ${ }^{97}$.

Du côté occidental, on assiste aussi à quelques réactions passionnées. Rappelons que Sigismond Pandolfe Malatesta est un admirateur de Pléthon, au point qu'à la suite d'une campagne militaire vénitienne contre les Turcs dans le Péloponnèse, en 1464-1465, il rapporte le corps de Pléthon et le fait déposer dans un sarcophage qui se trouve toujours sur un mur extérieur du „Tempio Malatestiano“. (Le pape Pie II disait que c'était non un temple de chrétiens, mais d'infidèles adorant des démons ${ }^{98}$.) Georges de Trébizonde, un grand ennemi de Pléthon, lie la mort de Sigismond Malatesta à la présence dans le „Tempio“, du corps de Pléthon et de l'Apollon qui vit dedans ${ }^{99}$ !

\section{Cosme de Médicis et les mages}

Mais revenons un peu en arrière, au concile de Ferrare-Florence sur l'union des Eglises (14381439). Pléthon qui y a participé à titre de conseiller laïque, a été amené à s'exprimer en marge du concile, sur un sujet à la mode en Italie, la supériorité de Platon sur Aristote (ou d'Aristote sur Platon $)^{100}$. Cosme de Médicis, selon Marsile Ficin, allait écouter Pléthon et en aurait conçu le projet „d'une sorte d'Académie““ ${ }^{101}$. Or, Pléthon a nécessairement rencontré Ambrogio Traversari au concile. Le camaldule avait traduit en latin les Vies et sentences des philosophes illustres de Diogène Laërce pour faire plaisir à Cosme et à Nicollò Niccoli. Il était surtout partisan d'un platonisme chrétien ${ }^{102}$. Mais Pléthon ne parle pas de Traversari. Il mentionne seulement Ugo [Benzi] et Pierre de Calabre (Pietro Vitali, abbé de Grottaferrata) ${ }^{103}$.

Qu'est-ce qui a pu intéresser Cosme qui ne parlait pas le grec et qui n'était pas un philosophe professionnel, dans ce que disait Pléthon, et qui était d'une grande technicité philosophique ? Ce

\footnotetext{
${ }^{97}$ Voir Tardieu/Nicolet 1980. L'édition critique de la traduction arabe des Oracles (La recension arabe des Magika logia) par Michel Tardieu, se trouve dans Pléthon 1995, 157-171.

${ }^{98}$ Piccolomini 1614, 51-52, texte cité par Masai 1956, 365, note 1; voir Bertozzi 2003a, 164 (et la note 41).

${ }^{99}$ Voir le texte et les références dans Monfasani 1976, 214 (et la note 85); voir aussi Garin 1989, 10; Bertozzi 2003b, 184-185.

${ }^{100}$ Voir Bertozzi 2003b, 180-181.

101 „Magnus Cosmus, senatus consulto patrie pater, quo tempore concilium inter Graecos atque Latinos sub Eugenio Ponifice Florentiae tractabatur, philosophum graecum, nomine Gemistum cognomine Plethonem, quasi Platonem alterum de mysteriis Platonicis disputantem frequenter audivit, e cuius ore ferventi sic afflatus est protinus, sic animatus, ut inde academiam quandam alta mente conceperit, hanc oportuno primum tempore pariturus". Ficin 1576, 1537. Je cite le texte latin collationné par James Hankins avec le manuscrit Plut. LXXXII, 10, conservé à la Biblioteca Laurenziana de Florence: voir Hankins 1970, 160 (Appendix II).

${ }^{102}$ La question de l'attitude de Traversari par rapport à la philosophie platonicienne est réexaminée par Arthur Field; je renvoie à sa communication: „Florentine Platonism before the Academy (ca. 1400-1450)“: voir Field 2010, cf. Hankins ${ }^{2}$ 1991, 64 (note 79), 59 et 130.

${ }^{103}$ Masai 1956, 334-336; 344; 358; Woodhouse 1986, 163-164.
} 
qui est certain c'est qu'ils avaient un intérêt commun pour les mages. Le Platon dont parle Pléthon est, comme Pythagore, l'héritier de la tradition des mages disciples de Zoroastre. Cosme de Médicis, pour sa part, avait trouvé chez les mages un modèle permettant de justifier aux yeux des Florentins, son activité de banquier international et la magie bancaire que constitue le prêt à intérêt. Il patronnait une confraternité, la Compagnia de' Magi qui organisait des processions pour la fête de l'Epiphanie (et de la Saint-Jean) au cours desquelles il défilait habillé en mage. Il avait fait représenter les mages dans sa cellule du couvent de San Marco où il faisait périodiquement des retraites. Il commanda à Benozzo Gozzoli la fresque du Voyage des mages pour décorer la chapelle de son palais de la Via Larga, voulant sans doute montrer que les Médicis assumaient l'héritage de la sagesse ancienne des mages, transmise aux Grecs, puis aux Latins. Comme je l'ai montré dans Pléthon. Le retour de Platon ${ }^{104}$ et dans „Pourquoi Cosme de Médicis a fait traduire Platon“"105, c'est sans doute cet intérêt bancaire pour les mages, et pour sa propre image, qui explique que Cosme de Médicis ait demandé à Marsile Ficin de traduire Platon - l'héritier des mages - en latin. De même, c'est pour les marchands, que Tommaso Benci traduit en 1463 Hermès Trismégiste (le Pimander) en italien, à partir de la traduction latine de Ficin, terminée quelques mois auparavant ${ }^{106}$.

Les Oracles magiques des mages disciples de Zoroastre ont eu du succès en Occident, puisque ce texte a été traduit plusieurs fois en latin avant d'être imprimé en grec ${ }^{107}$. Il existe par exemple une traduction latine des Oracles que S. Gentile attribue à Giano Lascaris (ca. 1445-1535) ${ }^{108}$. Marsile Ficin possède lui aussi la collection d'Oracles de Psellos et celle de Pléthon avec leurs commentaires respectifs, dans un manuscrit contenant plusieurs œuvres de Pléthon, l'actuel Riccardianus graecus 76, comme en témoigne l'index grec. Mais ces copies ont été détachées par la suite et on ne sait pas ce qu'elles sont devenues. Ficin traduit les vers en latin et les utilise en tant qu'autorité fondamentale, dans sa Théologie platonicienne, en incorporant aussi des morceaux du Commentaire de Pléthon et de l'Exegesis de Psellos ${ }^{109}$. Mais les Oracles sont désormais mis sous des autorités qui n'auraient pas du tout plu à Pléthon: les mages, Zoroastre, les Chaldéens et même les magiciens !

Pic de la Mirandole possédait lui aussi la collection des Oracles magiques des mages disciples de Zoroastre. Un mythe s'est forgé autour de sa copie, de sorte que lorsque Thomas Stanley redécouvre la collection à la fin du XVII ${ }^{\mathrm{e}}$ siècle, il croit que Pic possédait l'original des Oracles en langue chaldéenne, et Jean Le Clerc qui est arminien mais aussi très proche des idées néoariennes, répète la même information en français et la diffuse en latin à travers l'Europe ${ }^{110}$. Le propos, évidemment, inquiète les milieux ,orthodoxes“, dans le contexte des querelles trinitaires de la fin du XVII ${ }^{\mathrm{e}}$ siècle, et Pierre-Daniel Huet réplique en affirmant que la collection de Pléthon réimprimée en 1689 à Amsterdam, est un faux ! Bayle enregistre l'information dans son Dictionnaire, à l'article ,Zoroastre“.

Ce qui est intéressant, c'est que cette œuvre de philosophie versifiée que constituent les Oracles, est largement diffusée à la Renaissance et que ceux-ci sont considérés comme des Oracles „,de Zoroastre" qui redevient le maître des Chaldéens (il n'est plus aussi ancien que chez Plutarque bien qu'il fasse partie des prisci). Pour suivre la diffusion des Oracles, on dispose d'un

\footnotetext{
104 Tambrun 2006, 9-33.

${ }^{105}$ Voir Tambrun 2009.

${ }^{106}$ Voir les références dans Tambrun 2006, 30.

${ }^{107}$ L'editio princeps du texte grec, faite par Joannes Lodoicus Tiletanus (Jean Loys de Thielt) à Paris, date de 1538.

${ }^{108}$ Pléthon 1995, lxxii (note 9), Stausberg 1998, 127-129; voir aussi Seng 2010.

${ }^{109}$ Voir Tambrun 1999a et Tambrun 1999b.

${ }^{110}$ Voir Tambrun 2010.
} 
remarquable instrument de travail: le livre de Michael Stausberg, Faszination Zarathushtra. Zoroaster und die Europäische Religionsgeschichte der Frühen Neuzeit. En effet, en suivant les mentions du nom de Zoroastre dans la littérature religieuse ou philosophique de l'époque moderne, répertoriées par M. Stausberg, on retrouve facilement la collection des Oracles de Pléthon, dans la mesure où elle continue à être mise sous le nom de Zoroastre. Mais cette collection est aussi évolutive: Francesco Patrizi da Cherso (1529-1597) en organise une nouvelle ${ }^{111}$, à partir de celle de Pléthon, de Psellos, et des oracles qui se trouvent dans les œuvres des néoplatoniciens ${ }^{112}$.

\section{Postérité des Hymnes: Michel Marulle}

Les Hymnes en vers de Pléthon ont été pieusement recueillis et diffusés par ses amis et disciples. Il faut imaginer que l'interdiction, prononcée par le patriarche de Constantinople, de copier le reste du livre de Pléthon, jeté au feu, n'a pas découragé tous les partisans du philosophe, puisque des copies du reste du Traité des lois, et donc des Hymnes en vers, ont circulé en Italie dès le $\mathrm{XV}^{\mathrm{e}}$ siècle.

Michel Marulle (ca. 1453-1500), fils de réfugiés grecs, qui a fréquenté le cercle de Ficin et de Pic de la Mirandole, compose des Hymnes naturels ${ }^{113}$ aux dieux, qui font pour ainsi dire concurrence à ceux de Pléthon. Marulle était un soldat toujours prêt à prendre les armes et à partir en croisade pour délivrer sa patrie de la domination ottomane. Contrairement aux Hymnes de Pléthon, ceux de Marulle constituent véritablement une oeuvre de poésie, écrite en très beaux vers latins, qui inspirera d'ailleurs Pierre de Ronsard. La théologie païenne et néo-platonicienne des Hymnes de Marulle concorde désormais dans un esprit ficinien, avec le christianisme ${ }^{114}$. Ainsi, la poésie méprisée par Pléthon qui, refusant de „fuir d'ici-bas“"115, cherchait à restaurer l'hellénisme (et donc les sciences) et à l'ancrer dans son terroir, fait retour chez cet hellène émigré qui ne peut plus aspirer qu'à un retour dans une patrie céleste.

\section{Bibliographie}

\section{Quellen}

Ammonius (1895): In Aristotelis De interpretatione commentarius, Hrsg. von Busse, A., Berlin $(=$ C.A.G. IV , 2).

Aristide Quintilien [Aristides Quintilianus] (1963): De musica, Hrsg. von Winington-Ingram, R.-P., Leipzig: Teubner.

Aristide Quintilien (1999): La Musique, Übersetz. von Duysinx, F., Liège: Droz (= Bibliothèque de la faculté de philosophie et lettres de l'université de Liège).

Bryennios, Manuel (1970): The Harmonics of Manuel Bryennius, Hrsg. \& Übersetz. von Jonker, G.H., Groningen: Wolters-Noordhoff.

Euripide (1964): Phoenix, in: Tragicorum Graecorum Fragmenta, Hrsg. von Nauck, A., Hildesheim.

Ficin, Marsile (1576): Opera omnia, Bâle, Ndr. (1962), Turin.

\footnotetext{
${ }^{111}$ Patrizi 1593. Sur l'édition de cette collection, voir Stausberg 1998, 321-324.

${ }^{112}$ Voir Stausberg 1998, 291-395.

${ }^{113}$ Marulle 1995.

${ }^{114}$ La théologie de Marulle est ainsi très ambiguë: on ne saurait dire si les trois premiers dieux (Jupiter, Pallas, Amour) sont subordonnés les uns aux autres ou s'ils forment une Trinité.

${ }^{115}$ Cf. Platon, Théétète 176 a-b.
} 
Grégoras, Nicéphore (1975): Florentios ou de la sagesse: Niceforo Gregora, Fiorenzo o intorno alla sapienza. Hrsg., Übersetz. \& Komment. von P.L.M. Leone, Napoli: Università di Napoli.

Homeric Hymns, Homerica apocrypha, Lives of Homer (2003): Hrsg. \& Übersetz. von West, Martin L., London/Cambridge: Harvard University Press (= Loeb Classical Library, 496).

Marulle, Michel (1995): Hymnes naturels, Hrsg. von Chomarat, Jacques, Genève: Droz.

Métochite, Théodore (2007): Peri tou mathêmatikou eidous tês philosophias kai malista peri tou harmonikou, Hrsg. von Polemis, Ioannis, Amsterdam: A.M. Hakkert (= Classical and Byzantine monographs, 61).

Olympiodore (1970): In Platonis Gorgiam Commentaria, Hrsg. von Westerink, L.-G., Leipzig: Teubner.

Pachymère, Georges (1940), Quadrivium, Hrsg. von Tannery, Paul/Stéphanou, E., Città del Vaticano: Biblioteca Apostolica Vaticana (= Studi e testi, 94).

Patrizi, François (1593): Magia Philosophica, hoc est Francisci Patricii summi philosophi Zoroaster et eius 320 Oracula Chaldaica, Hamburg.

Philopon, Jean (1897): In Aristotelis De anima libros commentaria, Hrsg. von Hayduck, M., Berlin (= C.A.G., XV).

Photius (1967): Bibliothèque, Hrsg. von Henry, R., Bd. V (cod. 230-241), Paris: Les Belles Lettres.

Piccolomini, Enea Silvio (Pio II) (1614): Commentarium rerum memorabilium, Francfort.

Piccolomini, Enea Silvio (Pio II) (1984): I, Commentarii, Hrsg. von Totaro, L. Milan: Adelphi.

(Pseudo-) Plutarque (1996): Essay on the life and poetry of Homer, Hrsg. von Keaney, J.J./ Lamberton, Robert, Atlanta (Ga): Scholars Press (= American classical studies, 40).

Psellos, Michel: Commentaire des Oracles chaldä̈ques, in: des Places, Édouard (1971): Oracles chaldaïques avec un choix de commentaires anciens, Paris: Les Belles Lettres.

Psellos, Michel: Commentaire des Oracles chaldä̈ques, in: Psellus, Michael (1989): Philosophica minora, Hrsg. von O’Meara, D.J., Bd. II, Leipzig: Teubner, 126-146.

Pléthon [Gemistos], Georges (1858): Traité des lois, Hrsg. von Alexandre, C., [Übersetz. von Pellissier, A.], Paris. [La réédition de Brague, Rémi (1982), Paris: Vrin, est partielle.]

Pléthon [Gemistos], Georges (1926): Mémoire pour Manuel, in: Lambros, Spiridon (Hrsg.): Palaiologeia kai Peloponnêsiaka, Bd. III, Athens: Epitropê ekd. tôn Kataloipôn Spuridônos Lamprou, 246-265.

Pléthon [Gemistos], Georges (1930): Mémoire pour Théodore, in: Lambros, Spiridon (Hrsg.): Palaiologeia kai Peloponnêsiaka, Bd. IV, Athens: Epitropê ekd. tôn Kataloipôn Spuridônos Lamprou, 113-135.

Pléthon [Gemistos], Georges (1989): Réplique à Scholarios, Hrsg. \& Übersetz von Lagarde, Bernadette, in: „Georges Gémiste Pléthon: Contre les objections de Scholarios en faveur d'Aristote (Réplique)“, Byzantion 59, 354-507.

Pléthon [Gemistos], Georges (1987): Traité des vertus, Hrsg., Übersetz. \& Komment. von Tambrun-Krasker, Brigitte, Athens: The Academy of Athens/Leiden: Brill (= Corpus Philosophorum Medii Aevi, Philosophi Byzantini, 3).

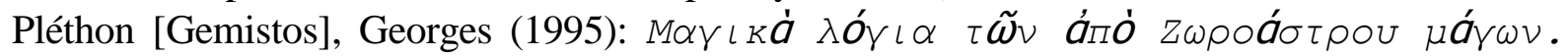

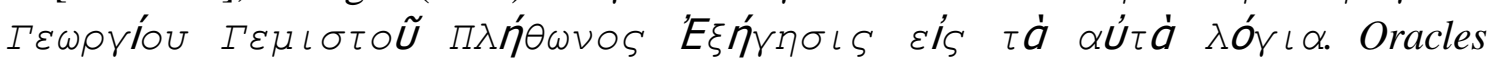
chaldä̈ques. Recension de Georges Gémiste Pléthon, Hrsg., Übersetz. \& Komment. von Tambrun-Krasker, Brigitte. La recension arabe des $M \alpha \gamma\llcorner$ kà $\lambda$ ó $\llcorner\alpha$, Hrsg., Übersetz. \& Komment. von Tardieu, Michel, Athens: The Academy of Athens/Paris: J. Vrin/Bruxelles: Ousia (= Corpus Philosophorum Medii Aevi, Philosophi Byzantini, 7). 
Pléthon [Gemistos], Georges (1998): Manuel d'astronomie, Hrsg. \& Übersetz. von Tihon, A./Mercier, R., Louvain-la-neuve: Bruylant-Academia (= Corpus des astronomes byzantins, 9$)$.

Porphyre (1998): Isagoge, Übersetz. De Libera, A./Segonds, A. -Ph., Paris: Vrin.

Pythagore (1962): I versi aurei di Pitagora, Hrsg. von Farina, A., Napoli (= Collana di studi greci, 35).

Proclus (1899-1901): In Rempublicam, Hrsg. von Kroll, W., Bd. I-II, Leipzig: Teubner (Ndr. (1965) Amsterdam).

Proclus (1968): Théologie platonicienne, Hrsg. \& Übersetz. von Saffrey, H.-D./Westerink, L.G., Bd. I, Paris: Les Belles Lettres.

Prolégomènes à la philosophie de Platon (1990), Hrsg. \& Übersetz. von Westerink, L.-G., Paris: Les Belles Lettres.

Ptolémée, Claude (1930): Die Harmonielehre des Klaudios Ptolemaios, Hrsg. von Düring, Ingmar, Göteborg.

Scholarios Gennade, Georges (1928-1936): Oeuvres complètes, Hrsg. von Petit, L./Sidéritès, X.A./Jugie, M., Bd. I-VIII, Paris: Maison de la bonne presse.

Simplicius (1990): Commentaire sur les Catégories, Traduction commentée sous la direction de I. Hadot, Fascicule I, Introduction, Première partie. Traduction de Ph. Hoffmann. Commentaire et notes à la traduction par I. Hadot, Leiden: Brill.

Suidas (1928, ${ }^{2}$ 1971): Suidae Lexicon, Hrsg. von Adler, A., Stuttgart: Teubner.

\section{Forschungsliteratur}

Bélis, Annie (1996): „Harmonique“, in: Brunschwig, Jacques/Lloyd, Geoffrey (Hrsg.): Le savoir grec. Dictionnaire critique, Paris: Flammarion, 352-367.

Bertozzi, Marco (2003a): „Segni, simboli, visioni: il Tempio Malatestiano e i suoi enigmi“, in: Musmeci, Marco (Hrsg.): Templum Mirabile (Atti del Convegno sul Tempio Malatestiano, Rimini, 21-22 settembre 2001), Rimini: Fondazione Cassa di Risparmio di Rimini, 151165.

Bertozzi, Marco (2003b): „George Gemistos Plethon and the Myth of Ancient Paganism: From the Council of Ferrara to the Tempio Malatestiano in Rimini“", in: Benakis, L.G./Baloglou, Ch. P. (Hrsg.): Proceedings of the International Congress on Plethon and his Time (Mystras 26-29 June 2002), Athens-Mystras: Société internationale d'études pléthoniennes et byzantines, 177-185.

Brechet, C. (2005): „Vers une philosophie de la citation poétique: écrit, oral et mémoire chez Plutarque“, in: Plutarch's Philosophical Tactics (Dublin, Trinity College, July 2005), Hermathena 182, 101-134.

Cacouros, Michel (1996): „Un manuel de logique organisé par Jean Chortasménos et destiné à l'enseignement", Revue des Études Byzantines, 54, 67-106.

Cacouros, Michel (1997): „Jean Chortasménos Katholikos didaskalos“, in: Criscuolo, U./Maisano, Riccardo (Hrsg.): Synodia, Napoli: M. d'Auria editore, 83-107.

Field, Arthur (2010): „Florentine Platonism before the Academy (ca. 1400-1450)“, in: „Platonismus und Esoterik in byzantinischem Mittelalter und italienischer Renaissance“, colloque international organisé par Helmut Seng, Frankfurt am Main, Goethe Universität, 19. bis 23. Juli 2010, (à paraître dans les actes du colloque).

Friedl, A.J. (1936): Die Homer-Interpretation des Neuplatonikers Proklos, Inaug. Dissert. Würzburg.

Fuchs, Friedrich (1926): Die höheren Schulen von Konstantinopel, Stuttgart, [Ndr. (1964), Amsterdam]. 
Buffière, F. (1956, ${ }^{2}$ 1973), Les mythes d'Homère et la pensée grecque, Paris: Les Belles Lettres. Couloubaritsis, Lambros (1997): „La métaphysique de Pléthon. Ontologie, théologie et pratique du mythe“, in: Neschke-Hentschke, Ada (Hrsg.): Images de Platon et lecture de ses oeuvres, (Lausanne)/Louvain/Paris: Peeters.

Des Places, Édouard (1971): Oracles chaldäques avec un choix de commentaires anciens, Paris: Les Belles Lettres.

Droge, A.J. (1989): Homer or Moses? Early Christian Interpretations of the History of Culture, Tübingen: Mohr.

Garin, E. (1989): Umanisti, artisti, scienzati. Studi sul Rinascimento italiano, Roma: Editori Riuniti.

Hankins, James (1970): „Cosimo de' Medici and the 'Platonic Academy'“, in: Journal of the Warburg and Courtauld Institutes, 33, 144-162.

Hankins, James ( $\left.{ }^{2} 1991\right)$ : Plato in the Italian Renaissance, Leiden/ New York/ Kobenhaven/ Köln: Brill (second impression with addenda et corrigenda).

Masai, François (1956): Pléthon et le platonisme de Mistra, Paris: Les Belles Lettres.

Mioni, E (1985): Codices Graeci Manuscripti Bibliothecae divi Marci Venetiarum, Volumen II, Thesaurus Antiquus, (Codices 300-625), Roma.

Monfasani, John (1976): George of Trebizond: A Biography and a Study of his Rhetoric and Logic, Leiden: Brill.

O’Meara, D.J. (1989): Michael Psellus, Philosophica minora, Bd. II, Leipzig: Teubner.

Pagani, Fabio (2009): „Damnata verba: censure di Pletone in alcuni codici platonici“, in: Byzantinische Zeitschrift, 102/1, 167-202.

Reynolds, L.D./Wilson, N.G. $\left({ }^{1} 1968,{ }^{2} 1974\right)$ : Scribes and Scholars: A guide to the Transmission of Greek and Latin Literature, Oxford: Oxford University Press.

Reynolds, L.D./Wilson, N.G. (1984, $\left.{ }^{2} 1988,{ }^{3} 1991\right)$ : D'Homère à Erasme. La transmission des classiques grecs et latins, Paris: CNRS.

Reznikoff, Iegor (1981): „Pour une écologie de la musique“, CoEvolution, 6 (automne), [Paris].

Reznikoff, Iegor (1988): „L'intonation juste et l'interprétation de la musique ancienne“, in: Meslanges pour le $X^{e}$ anniversaire du Centre de musique ancienne de Genève (1975-1985), Genève, 35-45.

Seng, Helmut (2010): „Der Kommentar des Psellos zu den Chaldaeischen Orakeln in lateinischer Übersetzung (Vat. lat. 3122 f.44r-57r)“, in: „Platonismus und Esoterik in byzantinischem Mittelalter und italienischer Renaissance“, colloque international organisé par Helmut Seng, Frankfurt am Main, Goethe Universität, 19. bis 23. Juli 2010, (à paraître dans les actes du colloque).

Stausberg, Michael (1998): Faszination Zarathushtra, Zoroaster und die Europäische Religionsgeschichte der Frühen Neuzeit, Berlin/ New York: De Gruyter.

Tambrun, Brigitte (1999a): „Marsile Ficin et le Commentaire de Pléthon sur les Oracles chaldaïques", in: Accademia. Revue de la Société Marsile Ficin, Premier Numéro, 9-42.

Tambrun, Brigitte (1999b): „Un exemple d'utilisation du Commentaire de Pléthon par Ficin: Le véhicule de l'âme, le pneuma et l'idole“, in: Accademia. Revue de la Société Marsile Ficin, Premier Numéro, 43-48.

Tambrun, Brigitte (2006): Pléthon. Le retour de Platon, Paris: Vrin.

Tambrun, Brigitte (2009): „Pourquoi Cosme de Médicis a fait traduire Platon“, in: Amir-Moezzi, M.A./Dubois, J.-D. (Hrsg.): Pensée grecque et sagesse d'Orient. Hommage à Michel Tardieu, Turnhout: Brepols (= Bibliothèque de l'École des Hautes Études, Sciences Religieuses, 142), 653-667.

Tambrun, Brigitte (2010): „Jean Le Clerc lecteur des Oracles de Zoroastre: enjeux philosophiques et théologiques“, in: „Platonismus und Esoterik in byzantinischem 
Mittelalter und italienischer Renaissance“, colloque international organisé par Helmut Seng, Frankfurt am Main, Goethe Universität, 19. bis 23. Juli 2010, (à paraître dans les actes du colloque).

Tardieu, Michel/ Nicolet, J. (1980): „Pletho arabicus. Identification et contenu du manuscrit arabe d'Istanbul", Topkapi Serai, Ahmet III 1896, Journal Asiatique, 268, 35-57.

Tardieu, Michel (1987): „Pléthon lecteur des Oracles“, in: Mêtis 2, 141-164.

Wersinger, Anne G. (2008): La sphère et l'intervalle. Le schème de l'Harmonie dans la pensée des anciens Grecs d'Homère à Platon, Grenoble: Jérôme Millon.

West, M.L. (1992): Ancient Greek Music, Oxford: Clarendon Press.

Woodhouse, C.M. (1986): George Gemistos Plethon, The last of the Hellenes, Oxford: Clarendon Press.

Zakythinos, D. (1953): Le despotat grec de Morée, Bd. I, Histoire politique, Bd. II, Vie et institutions, Athènes (Ndr. (1975), Londres: Variorum). 